Krasnov, Dmitry; Zikanov, Oleg; Boeck, Thomas:

Numerical study of magnetohydrodynamic duct flow at high Reynolds and Hartmann numbers

URN: $\quad$ urn:nbn:de:gbv:ilm1-2014210048

Published OpenAccess: September 2014

Original published in:

Journal of fluid mechanics. - Cambridge [u.a.] : Cambridge Univ. Press (ISSN 1469-7645). - 704 (2012), S. 421-446.

DOI: $\quad 10.1017 / \mathrm{jfm} .2012 .256$

URL: $\quad$ http://dx.doi.org/10.1017/jfm.2012.256

[Visited: 2014-08-27]

„Im Rahmen der hochschulweiten Open-Access-Strategie für die Zweitveröffentlichung identifiziert durch die Universitätsbibliothek IImenau."

"Within the academic Open Access Strategy identified for deposition by Ilmenau University Library."

„Dieser Beitrag ist mit Zustimmung des Rechteinhabers aufgrund einer (DFG-geförderten) Allianz- bzw. Nationallizenz frei zugänglich."

"This publication is with permission of the rights owner freely accessible due to an Alliance licence and a national licence (funded by the DFG, German Research Foundation) respectively."

\section{DFG}

Nationallizenzen 


\title{
Numerical study of magnetohydrodynamic duct flow at high Reynolds and Hartmann numbers
}

\author{
Dmitry Krasnov $^{1} \dagger$, Oleg Zikanov ${ }^{2}$ and Thomas Boeck ${ }^{1}$ \\ ${ }^{1}$ Fakultät für Maschinenbau, Technische Universität Ilmenau, Postfach 100565, 98684 Ilmenau, Germany \\ 2 Department of Mechanical Engineering, University of Michigan - Dearborn, 4901 Evergreen Road, \\ Dearborn, MI 48128-1491, USA
}

(Received 23 March 2012; revised 4 May 2012; accepted 25 May 2012; first published online 3 July 2012)

High-resolution direct numerical simulations are conducted to analyse turbulent states of the flow of an electrically conducting fluid in a duct of square cross-section with electrically insulating walls and imposed transverse magnetic field. The Reynolds number of the flow is $10^{5}$ and the Hartmann number varies from 0 to 400 . It is found that there is a broad range of Hartmann numbers in which the flow is neither laminar nor fully turbulent, but has laminar core, Hartmann boundary layers and turbulent zones near the walls parallel to the magnetic field. Analysis of turbulent fluctuations shows that each zone consists of two layers: the boundary layer near the wall characterized by small-scale turbulence and the outer layer dominated by large-scale vortical structures strongly elongated in the direction of the magnetic field. We also find a peculiar scaling of the mean velocity, according to which the reciprocal von Kármán coefficient grows nearly linearly with the distance to the wall.

Key words: magnetohydrodynamics, MHD turbulence, turbulence simulation

\section{Introduction}

The duct flow of an electrically conducting fluid with an imposed constant magnetic field is nearly optimal for analysing fundamental properties of turbulence in liquid metal magnetohydrodynamics (MHD) as well as implications for many technological processes, such as, for example, continuous casting of steel or self-cooled liquid metal blankets for nuclear fusion reactors. While geometrically simple, the flow presents the key effects: turbulence with mean shear and the Hartmann and Shercliff boundary layers at the walls, respectively, perpendicular and parallel to the magnetic field. The history of studies of the MHD duct flow is as old as liquid metal MHD itself (Hartmann 1937; Hartmann \& Lazarus 1937). Nevertheless, as discussed further in this section, little is known about the flow behaviour at high Reynolds $(R e)$ and Hartmann $(\mathrm{Ha})$ numbers. Two reasons can be named. One is the difficulty of flow visualization in the experiments, which have to be conducted with non-transparent metals. Another is that, until recently, high values of $R e$ and $H a$ were inaccessible in computations. This paper presents the results of our attempt to fill the gap via high-resolution numerical simulations.

$\dagger$ Email address for correspondence: dmitry.krasnov@tu-ilmenau.de 
Experimental studies of the MHD duct flow have been conducted for a straight duct by Hartmann \& Lazarus (1937), Brouillette \& Lykoudis (1967), Reed \& Lykoudis (1978) and others (see Branover 1978 for a review) and for a toroidal duct, in which the flow is driven via interaction between radial current and axial magnetic field (Baylis 1971; Moresco \& Alboussière 2004). The reported flow properties included the profiles of mean velocity and fluctuation intensity measured by potential or Pitot probes and the integral friction coefficient. A certain understanding of the flow transformation under the impact of a magnetic field has been achieved, although the imprecision of local velocity measurements and impossibility of detailed threedimensional visualization remain serious problems. The integral friction coefficient, on the contrary, can be measured reliably and accurately. Traditionally, the change in its behaviour that occurs when a certain curve on the $\mathrm{Ha}-\mathrm{Re}$ plane is crossed has been identified as an indication of transition to turbulence or laminarization of the flow. It should be stressed, however, that, since the friction in an MHD duct flow at large $\mathrm{Ha}$ is dominated by the Hartmann boundary layers, the observed change of the friction coefficient behaviour indicates laminarization or transition in these layers, rather than in the entire flow. This is confirmed by the remarkably good agreement between the transition threshold determined experimentally in a toroidal duct of square cross-section (Moresco \& Alboussière 2004) and the threshold found for the Hartmann boundary layers in the numerical study of Krasnov et al. (2004).

Another approach to the analysis of MHD flows with an imposed strong constant magnetic field is based on the quasi-two-dimensional approximation that assumes that the flow outside the Hartmann layers is nearly two-dimensional, i.e. nearly uniform along the magnetic field lines (Sommeria \& Moreau 1982; Potherat, Sommeria \& Moreau 2000; Potherat 2007). The analysis is valid at values of the magnetic interaction parameter $N \equiv \mathrm{Ha}^{2} / \mathrm{Re}$ much larger than one. It is possible that, in some systems, turbulence exists when $N \gg 1$ (Potherat \& Schweitzer 2011), but no examples of such turbulence in MHD channel or duct flows have been reported. In our study, $N$ is either smaller than or about one, so the quasi-two-dimensional behaviour is not expected and the approach is not applicable.

Numerical analysis of turbulence via direct numerical simulation (DNS) and large eddy simulation (LES) has been quite extensively applied to MHD flows in channels (see e.g. Lee \& Choi 2001; Kobayashi 2006; Satake et al. 2006; Boeck, Krasnov \& Zienicke 2007; Sarris, Kassinos \& Carati 2007; Krasnov et al. 2008) and ducts (see e.g. Burr et al. 2000; Kobayashi 2008; Chaudhary, Vanka \& Thomas 2010; Shatrov \& Gerbeth 2010). Fully turbulent as well as transitional flow regimes were investigated. Significant results were obtained concerning the effect of the magnetic field on structure and transport properties of the flow as well as the suitability of numerical models such as LES closures for MHD turbulence. Some of these results, which have direct relevance to our findings, are discussed further in the text.

Of particular interest for our study are the numerical simulations at high Reynolds numbers, i.e. for the case in which a magnetic field of significant strength has to be applied to laminarize the flow. For channel flow with a wall-normal magnetic field, the highest values of $R e$ were achieved in the DNS of Satake et al. (2006) and Boeck et al. (2007), where the Reynolds number based on the channel half-width and mean velocity was, correspondingly, 22909 and 21600 . In either study, the Hartmann number was not very large, so the flow remained fully turbulent. Turbulent channel flow with a spanwise magnetic field at $R e=13333$ was analysed in the DNS of Krasnov et al. (2008). The study most relevant to our work is the LES of Kobayashi (2008). A flow in a square duct with electrically insulating walls and a transverse 
magnetic field was computed at $R e=14500$ and 2650 and $H a$ up to 72.5. As will be discussed further in this paper, the results are in agreement with our results.

Until recently, two factors limited the Reynolds and Hartmann numbers in DNS studies. One was the lack of computational power sufficient for operating on the fine grids needed to resolve the small-scale turbulent structure and the thin Hartmann boundary layers. Another was the absence of robust computational schemes capable of dealing with nonlinear unsteady high-Ha flows. In the case of LES, Re could be somewhat increased, but the resolution requirements for the Hartmann layers remained essentially the same as in DNS, since no reliably accurate wall function models were known for the case of turbulent flows.

In the work reported in this paper, the obstacle of insufficient computational power is overcome via massively parallel computations. As a numerical method, we apply the finite difference scheme described by Krasnov, Zikanov \& Boeck (2011a). The scheme is based on the conservative discretization of Morinishi et al. (1998) extended to the case of MHD flows by Ni et al. (2007). We have found that the scheme is capable of accurate and numerically stable simulations of flows at high Hartmann number.

Several recent computational studies of MHD duct flow have direct relevance to the work presented in this paper. We have already mentioned the LES of Kobayashi (2008). Krasnov et al. (2010) studied the optimal modes (those with the strongest transient growth) of the laminar MHD duct flow and found that these modes resided within the sidewall layers of the duct. Since the growth and breakdown of these modes form the likely mechanism of transition to turbulence, the results suggest that the transition first occurs within the sidewall layers, while the flow remains laminar in the core of the duct and in the Hartmann layers. The suggestion has been confirmed in the recent DNS at moderate $\mathrm{Re}$ and $\mathrm{Ha}$ (Krasnov et al. 2011b). It has been found that, at a given $R e$, the flow state does not change directly from fully turbulent to fully laminar or vice versa when the Hartmann number is, respectively, increased or decreased. There is a range of $\mathrm{Ha}$ in which the flow has laminar core and Hartmann layers and two or one turbulent sidewall layers. Flow regimes with a laminar core and turbulence in the sidewall layers were also detected at $R e=14500$ and $H a=72.5$ in the LES of Kobayashi (2008). A qualitatively similar result was found by Zhao \& Zikanov (2012). In a toroidal duct flow with a strong axial magnetic field, the sidewall layer near the outer cylindrical wall becomes turbulent at much smaller Reynolds numbers than the rest of the flow.

In this paper we report the results of high-resolution DNS of MHD duct flow at high Reynolds and Hartmann numbers. The main distinction from the previous studies of the subject is, apart from much higher $R e$ and $H a$, the detailed analysis of the internal structure of velocity and electric current fields, which is conducted for $\mathrm{Ha}$ ranging from zero to the values at which the flow becomes fully laminar.

\section{Physical model and numerical method}

We consider a flow of an electrically conducting, incompressible, Newtonian fluid, e.g. a liquid metal, in a duct of square cross-section and side $2 d$ schematically shown in figure 1. The flow is driven by an applied streamwise pressure gradient, which is adjusted in the numerical model so that the mean velocity $U_{q}$ remains constant. A uniform constant magnetic field $B \boldsymbol{e}_{z}$ is imposed so that the magnetic field lines are parallel to one set of walls (the sidewalls, or Shercliff walls) and perpendicular to the other walls (the Hartmann walls). The walls of the duct are assumed to be solid, 


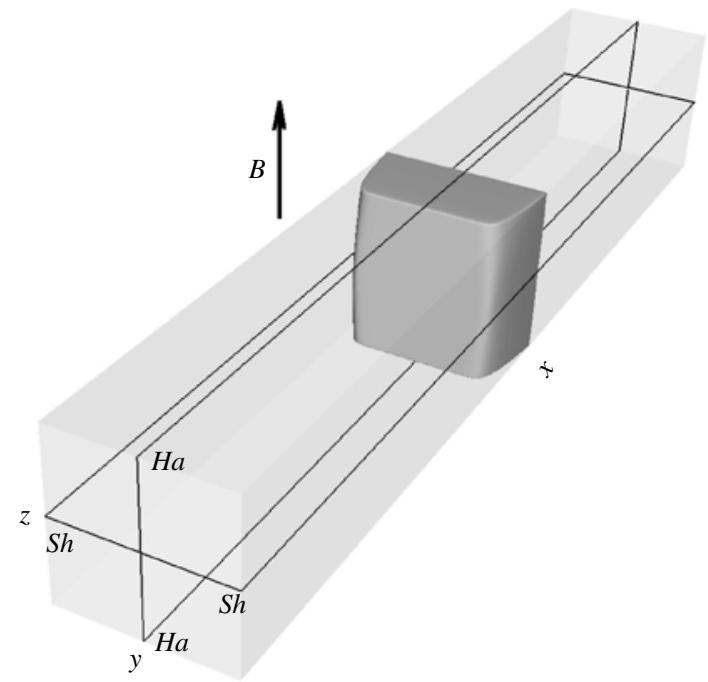

FIgURE 1. Flow geometry. The mid-plane cross-sections along $(H a-H a)$ and across $(S h-S h)$ magnetic field, extending, respectively, between the opposite Hartmann and Shercliff walls, will be used for flow analysis and visualization.

smooth, and perfectly electrically insulating. At the inlet and exit of the computational domain, periodic boundary conditions are imposed.

We assume that the magnetic Reynolds number $R e_{m} \equiv U_{q} d / \eta$ and the magnetic Prandtl number $\operatorname{Pr}_{m} \equiv v / \eta$ are both much smaller than one. Here, $v$ is the kinematic viscosity and $\eta=\left(\sigma \mu_{0}\right)^{-1}$ is the magnetic diffusivity of the fluid, $\sigma$ and $\mu_{0}$ being its electric conductivity and the magnetic permeability of vacuum. The assumption means that the fluctuations of the magnetic field caused by the velocity fluctuations are much smaller than the imposed magnetic field and have negligibly small adjustment time. The quasi-static approximation of the MHD equations (see e.g. Davidson 2001) can therefore be applied.

A comment is in order regarding the validity of the quasi-static approximation in our case. At the hydrodynamic Reynolds number

$$
R e \equiv \frac{U_{q} d}{v}
$$

equal to $10^{5}$, the magnetic Reynolds number $\operatorname{Re}_{m}=\operatorname{RePr}_{m}$ is not very small for real liquid metals. At typical operating temperatures, $P r_{m}$ ranges approximately from $10^{-7}$ (mercury) to $10^{-5}$ (liquid sodium). As an example, the eutectic alloy GaInSt often used in liquid metal experiments has $\operatorname{Pr}_{m} \approx 1.4 \times 10^{-6}$. We consider the quasi-static approximation valid in our case because, as shown in the numerical analysis of MHD turbulence by Knaepen, Kassinos \& Carati (2004), deviations from the quasi-static behaviour are not significant when $R e_{m}$ is smaller, but not much smaller than one, or even about one.

The problem is formulated as non-dimensional using the following typical scales: the duct half-width $d$ for length, the mean velocity $U_{q}$ for velocity, $\rho U_{q}^{2}$ for pressure, the imposed field $B$ for magnetic field, $U_{q} B d$ for electric potential, and $\sigma U_{q} B$ for 
electric current density. The governing equations and boundary conditions are:

$$
\begin{gathered}
\frac{\partial \boldsymbol{u}}{\partial t}+(\boldsymbol{u} \cdot \nabla) \boldsymbol{u}=-\nabla p+\frac{1}{R e} \nabla^{2} \boldsymbol{u}+N\left(\boldsymbol{j} \times \boldsymbol{e}_{B}\right), \\
\boldsymbol{j}=-\nabla \phi+\left(\boldsymbol{u} \times \boldsymbol{e}_{B}\right), \\
\nabla \cdot \boldsymbol{u}=0, \\
\nabla^{2} \phi=\nabla \cdot\left(\boldsymbol{u} \times \boldsymbol{e}_{B}\right), \\
\boldsymbol{u}=0 \quad \text { at } z= \pm 1 \text { and } y= \pm 1, \\
\frac{\partial \phi}{\partial n}=0 \quad \text { at } z= \pm 1 \text { and } y= \pm 1,
\end{gathered}
$$

where $\boldsymbol{u}=(u, v, w), p, \boldsymbol{j}$ and $\phi$ are, correspondingly, velocity, pressure, current density and electric potential.

The non-dimensional parameters of the problem are the hydrodynamic Reynolds number (2.1) and the Hartmann number

$$
H a \equiv B d\left(\frac{\sigma}{\rho v}\right)^{1 / 2}
$$

or the magnetic interaction parameter

$$
N \equiv \frac{H a^{2}}{R e}=\frac{\sigma B^{2} d}{\rho U} .
$$

The numerical model is based on the explicit projection-type finite difference scheme of second order described as the scheme B in Krasnov et al. (2011a). The scheme uses the collocated grid arrangement and follows the principles developed by Morinishi et al. (1998) and extended to liquid metal MHD by Ni et al. (2007). It is nearly fully conservative in the sense that the numerical solution conserves mass, momentum and electric charge exactly, while the kinetic energy has a purely dissipative error of third order. The error can be tolerated due to its higher order, and even considered beneficial since its dissipative character means prevention of artificial growth of kinetic energy, and therefore preservation of numerical stability. The Poisson equations for pressure and electric potential are solved using the Fourier expansion in the streamwise $x$-direction and a direct cyclic reduction solver (Adams, Swarztrauber $\&$ Sweet 1999) in the $y-z$ plane.

Versions of the model have been applied in our recent simulations of transitional high- $\mathrm{Ha}$ flows in toroidal channel and duct (Zhao, Zikanov \& Krasnov 2011; Zhao \& Zikanov 2012) and of MHD turbulent channel flows with scalar transport (Dey \& Zikanov 2012).

The computational grid is structured and Cartesian. It is uniform in the streamwise direction and non-uniform in the duct cross-section, with the grid step reduced toward the walls of the duct. After experimenting with various grid stretchings, we have found that the optimal choice in our case is the distribution (the same along the $y$ - and $z$-axes)

$$
z(i)=\beta z_{\text {cheb }}(i)+(1-\beta) z_{\text {unif }}(i),
$$

where $i$ is the grid point index. The grid blends the Chebyshev Gauss-Lobatto stretching formula

$$
z_{c h e b}(\zeta)=\sin \left(\frac{\pi}{2} \zeta\right)
$$




$\begin{array}{ccccc}R e & H a & L_{x} \times L_{y} \times L_{z} & N_{x} \times N_{y} \times N_{z} & \begin{array}{c}\text { Grid factor } \\ \beta\end{array} \\ 100000 & \begin{array}{c}0,100,200,300,350, \\ 400\end{array} & 4 \pi \times 2 \times 2 & 2048 \times 769 \times 769 & 0.96\end{array}$

TABLE 1. Parameters of numerical simulations.

and the uniform distribution

$$
z_{\text {unif }}(\zeta)=\zeta
$$

where $-1 \leqslant \zeta \leqslant 1$ is the transformed coordinate in which the grid step is constant. The blending factor $\beta$ is taken to be 0.96 in our computations.

One advantage of the grid is that it avoids excessively large steps in the middle of the domain typical, for example, for the tanh-type stretchings. At the same time, the uniform grid component, while changing the grid only slightly in comparison with the pure Chebyshev Gauss-Lobatto distribution, results in a substantial increase of the numerical stability limit on the time integration step $\Delta t$. We have also found that the uniform grid component eliminates the problem of ill-conditioned matrices for the discrete Laplace operator in the Poisson equations for pressure and potential. The problem may appear as deterioration of precision in the case of strong clustering and large grid size in either the $y$ - or $z$-directions.

The parameters of the computational model are summarized in table 1. The single value $R e=10^{5}$ is used. This value can be compared, for example, with the simulations of the hydrodynamic channel flow by Hoyas \& Jiménez (2006), where Re was $\sim 50000$ in our units. To our knowledge, no simulations at such a high $R e$ have been performed for the duct. The Hartmann number varies from zero (the hydrodynamic case) to $H a=400$, at which the flow becomes fully laminar.

The computational domain has the streamwise dimension of $4 \pi$. This would be considered small in computations with moderate $R e$, but appears sufficient in our case due to the typically small size of turbulent structures expected at such a high $R e$ and confirmed by our flow visualizations discussed in $\S 3$.

The computational grid used to generate the results presented in this paper has 2048 uniformly distributed grid points in the streamwise direction and 769 points distributed according to (2.10) in each of the transverse directions. The time integration step is $\Delta t=10^{-3}$.

The following information provides the basis for assessing the quality of the grid. The streamwise grid step is $\Delta x \approx 0.0061$. In the wall-normal cross-section of the duct, the smallest grid step (nearest to the wall) is $\Delta y_{\min }=\Delta z_{\min } \approx 1.12 \times 10^{-4}$, while the largest step (in the middle of the duct) is $\Delta y_{\max }=\Delta z_{\max } \approx 4.03 \times 10^{-3}$. We can also evaluate the wall-normal resolution of boundary layers by measuring the grid steps in the wall units. We use the wall-friction Reynolds number

$$
R e_{\tau}^{S h}=\left.\frac{\partial U(y, z=0)}{\partial y}\right|_{y= \pm 1}, \quad R e_{\tau}^{H a}=\left.\frac{\partial U(y=0, z)}{\partial z}\right|_{z= \pm 1}
$$

calculated at the mid-plane sections $S h-S h$ and $H a-H a$ of the duct (see figure 1) using the time- and streamwise-averaged mean velocity $U(y, z)$ to derive the wall coordinates

$$
y^{+}=R e_{\tau}^{S h} y, \quad z^{+}=R e_{\tau}^{H a} z .
$$


The value of the smallest wall-normal grid step in the $z^{+}$units varies from 0.477 at $H a=0$ to 0.726 at $H a=400$. The respective variation in the $y^{+}$units is from 0.477 at $H a=0$ to 0.173 at $H a=400$ (see table 2 for the values of $R e_{\tau}^{H a}$ and $R e_{\tau}^{S h}$ at various $\mathrm{Ha}$ ).

A preliminary verification of the model was conducted by reproducing the results of Galletti \& Bottaro (2004) for the transient growth in the hydrodynamic duct and the results of Boeck et al. (2007) and Krasnov et al. (2008) for turbulent MHD flows in channels. We have also conducted a grid sensitivity study. At $R e=10^{5}$, we applied grids of stretching (2.10) and various sizes to compute the wall friction Reynolds numbers (2.13). In the flow with $H a=0$, the values of $R e_{\tau}^{S h}$ and $R e_{\tau}^{H a}$ computed on our final grid differ from the values computed on the cruder grid with $1024 \times 385^{2}$ points by less than $3 \%$. This shows reasonably good accuracy, but indicates that the DNS results reported in this paper for $H a=0$ are based on a slightly under-resolved model in which the numerical dissipation of the finite difference scheme provides a weak but not entirely negligible sink of kinetic energy.

The accuracy is higher for the flows with magnetic field. The difference in $R e_{\tau}$ between the results obtained on the final and cruder grids decreases below $1 \%$ at $H a=300$ and $H a=350$. As a further argument for the accuracy of the numerical model, we mention that the grid provides sufficient resolution of the Hartmann boundary layers. In the computations at $H a=400$ when the Hartmann layers are the thinnest, 26 grid points are located within the zone of the mean velocity increasing from zero to $95 \%$ of its centreline value.

The numerical experiments are conducted in a standard fashion. At each new value of $\mathrm{Ha}$, the flow is computed until it reaches a fully developed state, as indicated by statistically steady values of $R e_{\tau}^{S h}, R e_{\tau}^{H a}$, and other integral characteristics. After that, the flow is computed for the period not shorter than 28 convective time units, during which the time-averaged quantities are accumulated.

The large size of the computational grid and the large number of the needed time steps require a number of numerical operations quite big even by modern standards. To make this possible, we employ massive parallelization based on the hybrid approach with MPI (distributed memory) and OpenMP (shared memory) programming interfaces. The computations were conducted on the Juropa supercomputer at the Jülich Research Centre. Each production run has utilized 3072 computational cores. The total computational cost is $\sim 740$ thousands CPU-hours.

\section{Results}

The main integral characteristics of the computed, fully developed flows are listed in table 2. The characteristics for the laminar flows computed at the same $\mathrm{Ha}$ and $R e$ via artificial suppression of $u_{y}$ and $u_{z}$ and imposing flow uniformity in the streamwise direction are shown for comparison. The mechanisms and features of the flow determining the characteristics will be discussed further in this section.

\subsection{Flow structure at different strengths of the magnetic field}

We begin the discussion by presenting the general structure of the flow. Figure 2 shows the instantaneous distributions of the streamwise velocity $u$ in fully developed flows at four different values of $H a$. We see that already at $H a=100$, the flow develops a sizeable laminar core. The zones near the Shercliff and Hartmann walls are turbulent. At $H a=200$, turbulence remains in wide areas near the Shercliff walls. Outside these areas, the core and the flow near the Hartmann walls appear laminar. 


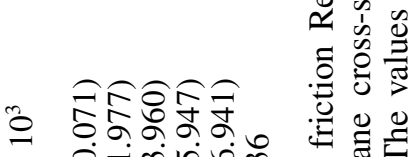

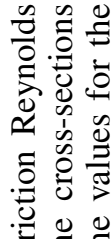

$x$ ejmine

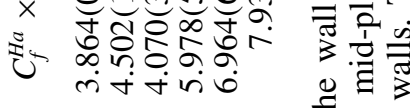

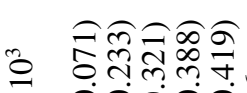

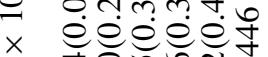

离

果

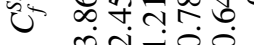

(

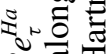

$\simeq$ त

ซี

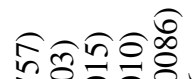

둥.

ज造

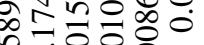

彳ी००:0:0:

$x^{2}$

늘

要的

$8 \%$

范 范

츠ำส

ฮี สิ

के

Ð

क के

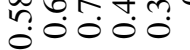

กิสิิสิ์

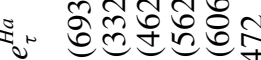

$\approx$ mon

भुजिए

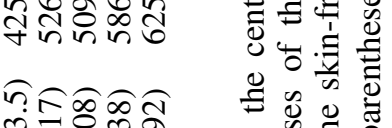

正

글.

응

记

的.

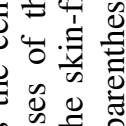

๘

†

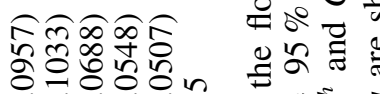

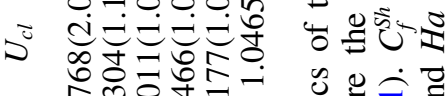

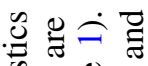

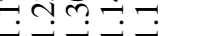

क्षे

z o

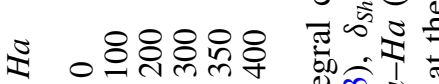

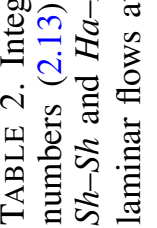


(a)

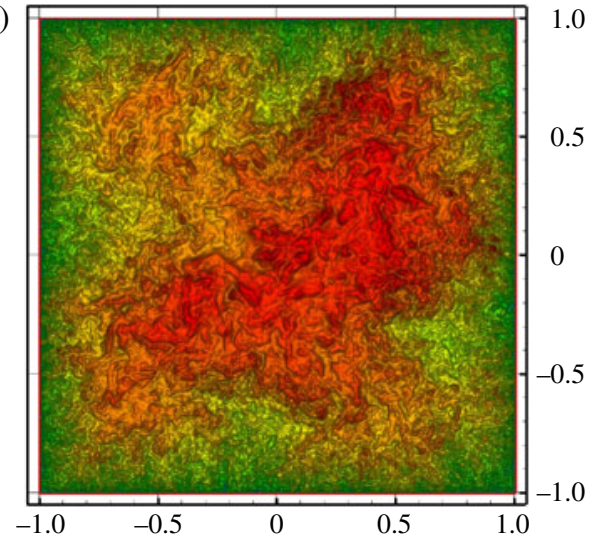

(c)

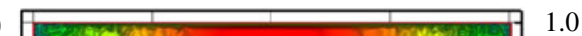

(b)

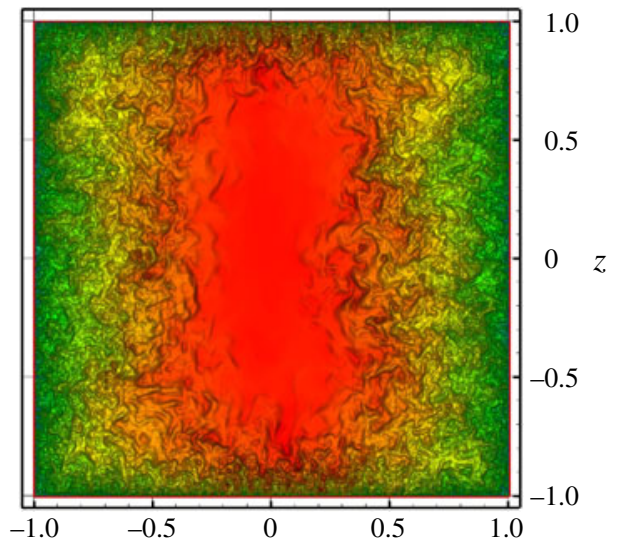

(d)

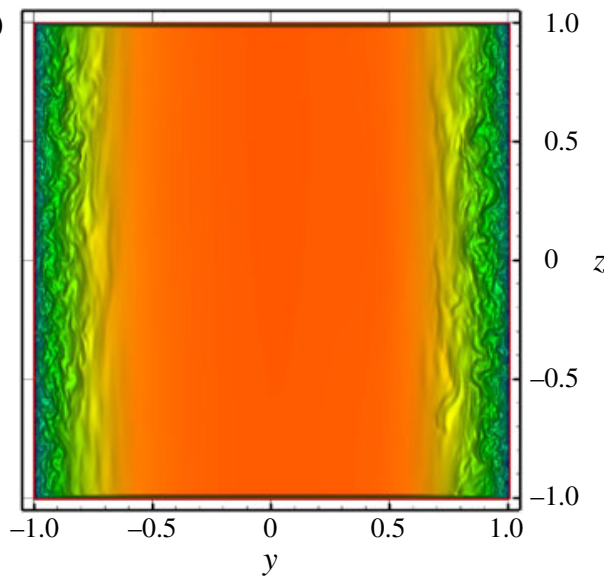

FIGURE 2. Instantaneous distributions of streamwise velocity at $x=L_{x} / 2$. The contour levels are the same in all plots ranging from 0 (blue) to 1.25 (red). Note that the levels corresponding to blue are virtually invisible due to the very sharp velocity gradients at the walls: (a) $H a=0$; (b) Ha= 100; (c) $H a=200 ;(d) H a=300$.

The analysis of the quantitative characteristics of turbulent fluctuations, such as the kinetic energy and the r.m.s. fluctuation profiles presented in $\S 3.3$, confirm that the laminarization is almost (but, as we demonstrate further, not entirely) complete.

Further increase of the Hartmann number leads to an increase of the laminar zone. As illustrated in figure 2, the turbulent layers become thinner at $H a=300$. Their thickness further decreases at $H a=350$ (not shown). At $H a=400$, the entire flow becomes laminar.

A qualitatively similar flow transformation at increasing $\mathrm{Ha}$ was found in the LES of Kobayashi (2008) performed at $R e=14500$. Visualization of coherent structures showed turbulence localized in the Shercliff layers at $H a=43.5$ and 58, while the flow became fully laminar at $\mathrm{Ha}$ equal to 65.25 .

The instantaneous distributions of electric currents obtained in our computations in the $y-z$ plane at $x=L_{x} / 2$ are shown in figure 3 . The fields correspond to the velocity fields at $H a=100$ and $H a=300$ in figure 2. One can see that in the laminar flow zone, where the velocity is almost entirely in the streamwise component, the current loops close in the $y-z$ plane. At $H a=300$ they have a structure qualitatively similar to 

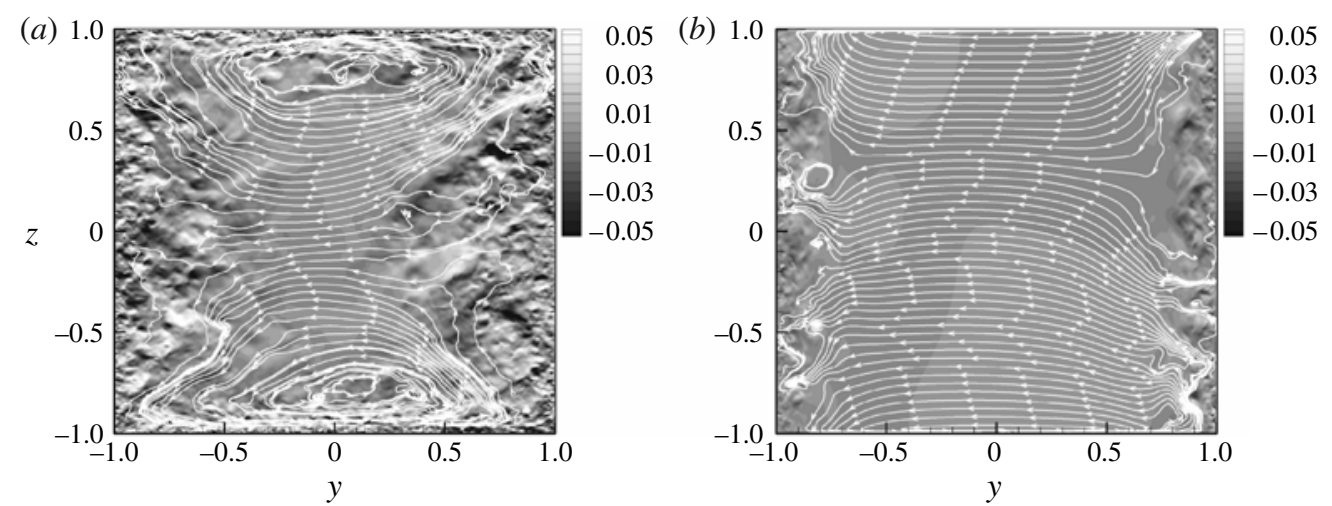

FIGURE 3. Instantaneous distributions of electric current density in the plane $x=L_{x} / 2$. Contours of the streamwise component $j_{x}$ and projection of streamlines passing through 50 uniformly distributed points on the line $y=0$ (the cross-section $\mathrm{Ha}-\mathrm{Ha}$ in figure 1) are shown: (a) $H a=100 ;(b) H a=300$.

the structure in a laminar duct flow (see e.g. Müller \& Bühler 2001). Currents flowing through the Hartmann boundary layers close within the core flow. In the turbulent zones near the Shercliff walls, where all three velocity components have non-negligible amplitude, $j_{x}$ is significant and the current loops are essentially three-dimensional.

\subsection{Mean flow}

The mean flow velocity is obtained as $\boldsymbol{U}=\langle\boldsymbol{u}\rangle$, where $\langle\cdots\rangle$ stands for averaging of a fully developed flow over time and the streamwise coordinate $x$. The results for the streamwise component $U(y, z)$ are shown in figure 4 in the form of two-dimensional distributions and in figure 5 using profiles in the mid-plane cross-sections $\mathrm{Ha}-\mathrm{Ha}$ and Sh-Sh (see figure 1).

An evident effect of the magnetic field is the suppression of flow variation along the magnetic field lines. The mean flow becomes nearly $z$-independent outside the boundary layers near the Hartmann walls.

The effect of the magnetic field on the mean velocity distribution along the $y$-axis is less trivial. We see that when the Hartmann number increases from 0 to 100 and then to 200, the centreline velocity increases (see figures 4 and 5 and table 2). The distribution takes a form similar to the chisel-like form found earlier in the simulations of channel flow with a spanwise magnetic field (Krasnov et al. 2008). This has been attributed to reduction of the wall-normal turbulent transport of momentum caused by the suppression of turbulence by the magnetic field. Our results presented in $\S 3.3$ support this explanation.

The tendency changes as the Hartmann number increases further. We see that the centreline velocity becomes smaller at $H a=300$ and 350 . This can be explained by development of a wide laminar core, where the flow begins to resemble the flat core of a laminar duct flow at high $\mathrm{Ha}$.

In order to analyse the boundary layer structure, we have measured the $95 \%$ thicknesses $\delta_{H a}$ and $\delta_{S h}$ of the Hartmann and Shercliff layers. The thicknesses are defined as the distances taken along the corresponding mid-plane cross-sections from the wall to the point where the mean velocity first exceeds $0.95 U_{c l}$. The results are shown in table 2 together with the thicknesses obtained for the laminar flows at the same $\mathrm{Re}$ and $\mathrm{Ha}$. In the laminar flows, the thicknesses follow very closely 
(a)

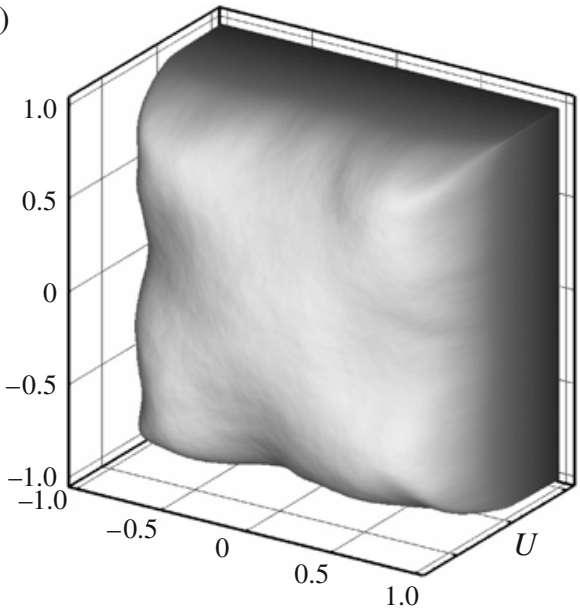

(c)

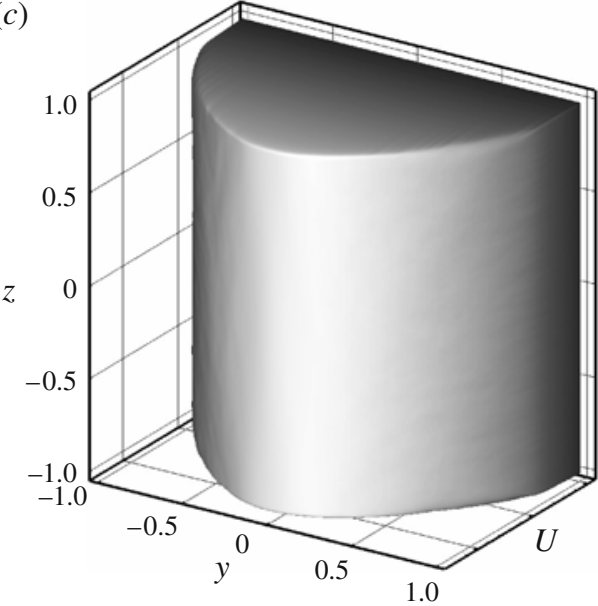

(b)

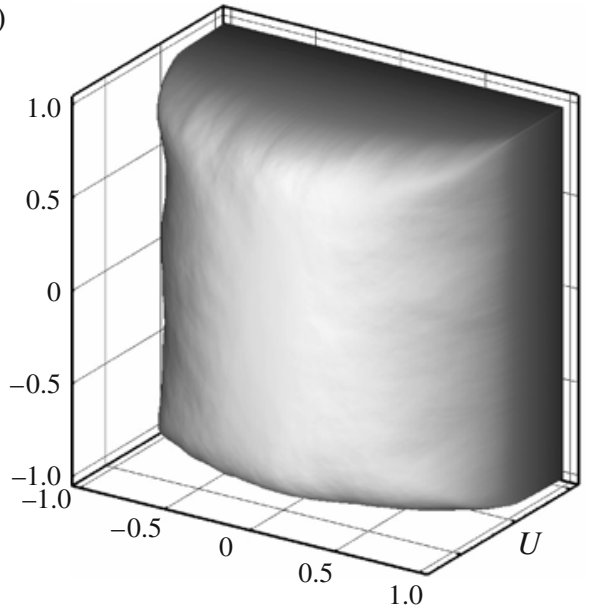

(d)

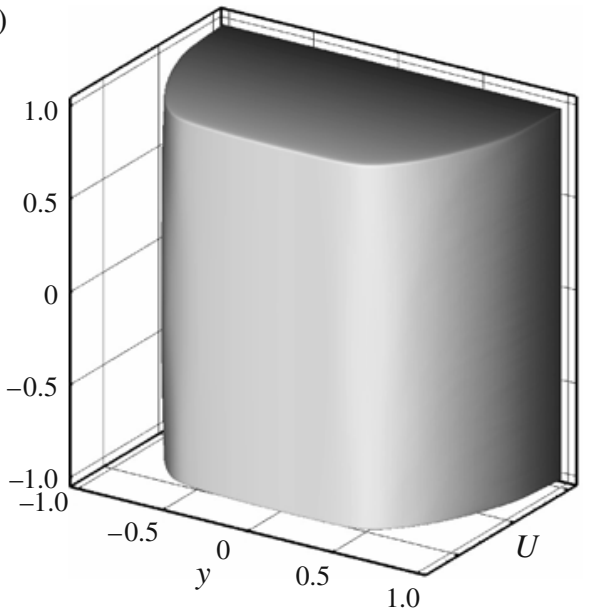

FIGURE 4. Distributions of the mean streamwise velocity $U(y, z)$. The contour levels are the same in all plots ranging from 0 to 1.25: (a) $\mathrm{Ha}=0$; (b) $\mathrm{Ha}=100 ;(c) H a=200 ;(d)$ $H a=300$.

the theoretical scaling $\sim d \mathrm{Ha}^{-1}$ for the Hartmann layers and $\sim d \mathrm{Ha}^{-1 / 2}$ for the Shercliff layers (see e.g. Müller \& Bühler 2001). In the real flow, the thicknesses are approximately the same when the corresponding layers near the walls are laminar. This is observed for the Hartmann layers at $H a \geqslant 200$ and for the Shercliff layers in the fully laminar flow at $\mathrm{Ha}=400$. If, however, the near-wall layers are turbulent, the measured thicknesses are much larger, so that no actual boundary layer behaviour can be claimed. Furthermore, the thickness of the sidewall layer changes nonmonotonically with $\mathrm{Ha}$. We can conclude that the classical picture of thin Hartmann and Shercliff boundary layers is inapplicable in the case of a not fully laminarized duct flow.

The transverse velocity components $V(y, z)$ and $W(y, z)$ of the mean flow are shown in figure 6. In the hydrodynamic case, we see a structure similar to those observed at smaller $R e$, for example, by Gavrilakis (1992) and Galletti \& Bottaro (2004). The secondary structures form a slightly distorted pattern of eight counter-rotating vortices 

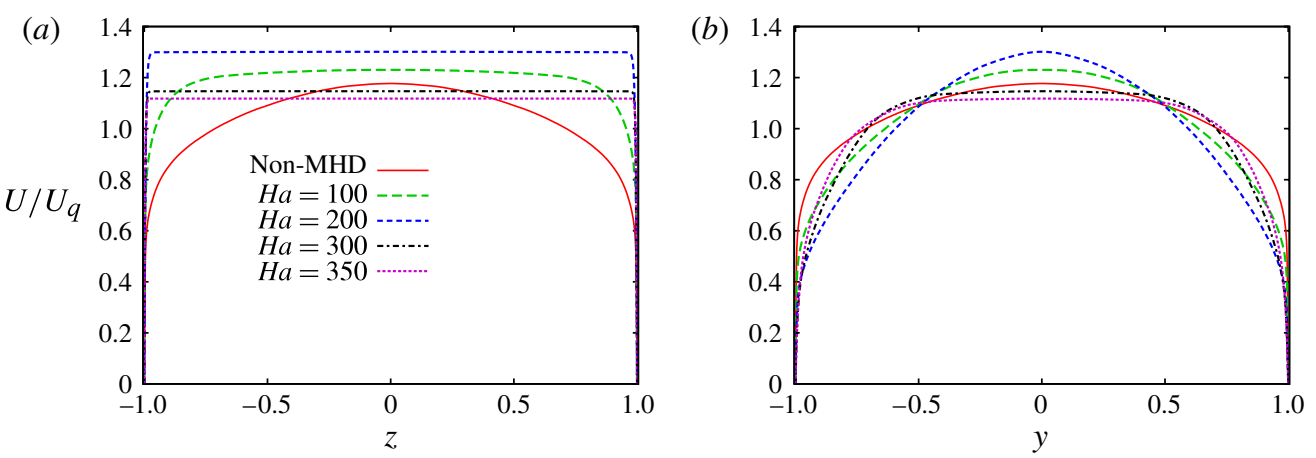

FIgURE 5. (Colour online) Profiles of mean flow velocity $U(y, z)$ in the cross-sections $(a)$ $\mathrm{Ha}-\mathrm{Ha}$ and $(b) \mathrm{Sh}-\mathrm{Sh}$.

in the corners of the duct. In the presence of the magnetic field, the secondary flow becomes weaker and loses its diagonal symmetry. At strong magnetic fields, when the flow develops a laminar core extending from one Hartmann wall to another, the mean secondary flow is limited to the turbulent zones near the Shercliff walls.

At this point we mention the results of computations by Uhlmann et al. (2007) obtained for a marginally turbulent hydrodynamic duct flow. They show that the structure of the secondary mean velocity field is sensitive to the time of averaging. The fully symmetric eight-vortex pattern similar to the pattern at $H a=0$ in our figure 6 is obtained by Uhlmann et al. (2007) as a definitive solution after averaging over 1700 convective time units. Other patterns, for example with four vortices, are found at smaller averaging time.

The much higher Reynolds number in our case implies smaller length and time scales of velocity fluctuations and so a smaller required averaging time. Nevertheless, the averaging time of 30 convective units used to produce the plots in figure 6 can be considered as a possible source of uncertainty. Another indication of somewhat insufficient averaging time is the imperfect symmetry of the vortex pattern at $H a=0$. The uncertainty diminishes in flows with high $\mathrm{Ha}$, since the magnetic field removes the diagonal symmetry and thus reduces the number of geometrically possible patterns.

The distributions of the time- and streamwise-averaged electric current density are shown in figure 7. Since the current is a linear function of velocity, the distributions present the currents that would be generated by the mean flow shown in figures 4 and 6. In the laminar core, the current loops are two-dimensional, lying in the $y-z$ plane. At $H a=300$, when the laminar core extends to the Hartmann walls, we see the classical feature of the entire core current closing within the Hartmann layers. In the turbulent zones, the vortices of the mean flow generate currents in the streamwise direction.

\subsection{Turbulent fluctuations}

The velocity fluctuation fields are computed as $\boldsymbol{u}^{\prime}=\boldsymbol{u}-\langle\boldsymbol{u}\rangle$, where $\langle\boldsymbol{u}\rangle$ is the mean flow velocity. In order to quantify their transformation under the influence of the magnetic field, we have computed the root-mean-square values shown in figure 8 . The figure also presents the Reynolds stress components $\tau_{12}=\left\langle u^{\prime} v^{\prime}\right\rangle$ and $\tau_{13}=\left\langle u^{\prime} w^{\prime}\right\rangle$ measuring the turbulent transport of momentum in the direction normal to, respectively, Shercliff and Hartmann walls. 

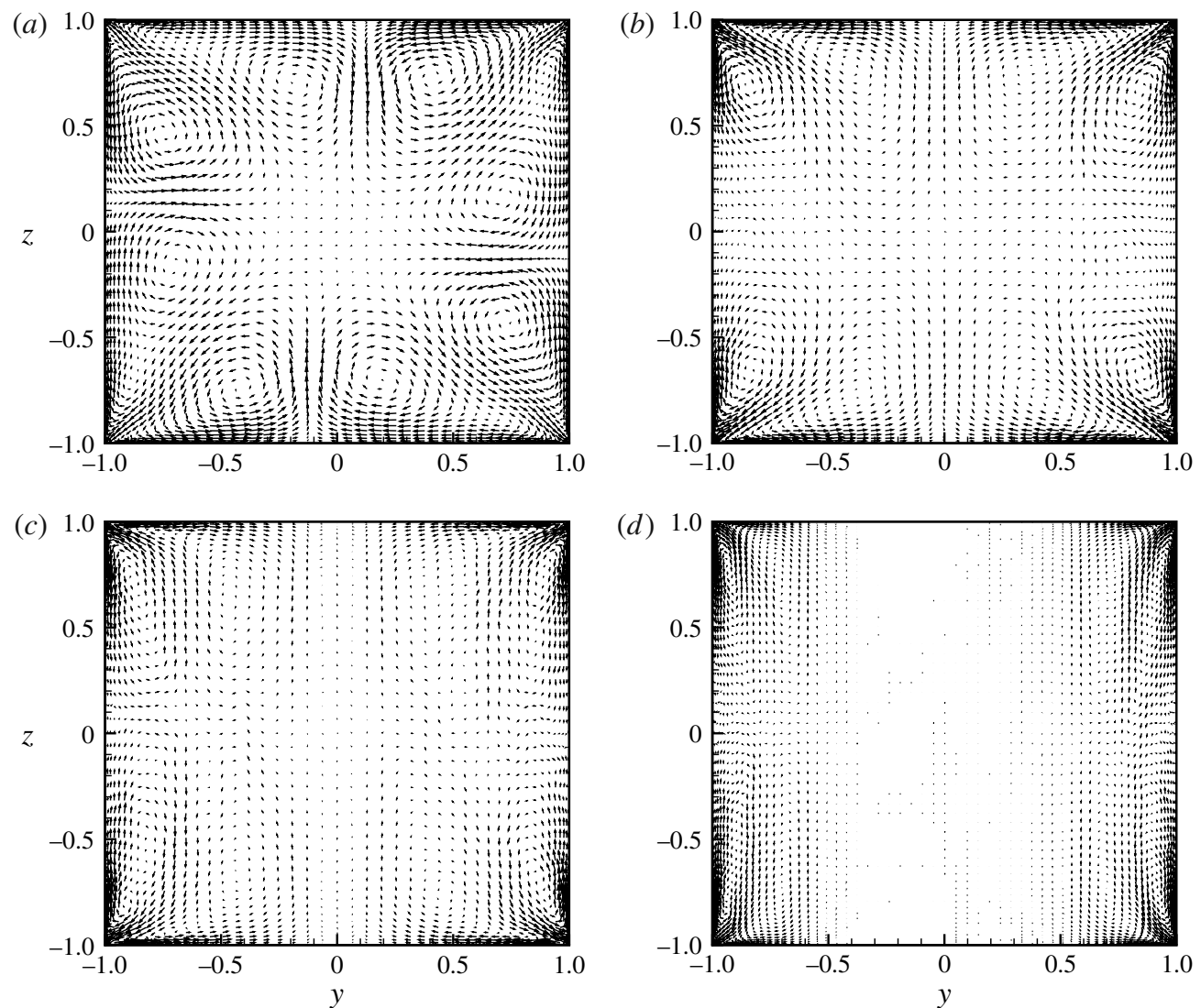

FIGURE 6. Distributions of the transverse velocity in the mean flow. Note that the amplitude of secondary motion decreases at high $\mathrm{Ha}$ due to the suppression of fluctuations by the magnetic field. To make the patterns visible, the vectors are scaled. The scaling factor is 3 at $H a=0,6$ at $H a=100$, and 12 at $H a=200,300:$ (a) $H a=0 ;(b) H a=100 ;$ (c) $H a=200$; (d) $\mathrm{Ha}=300$.
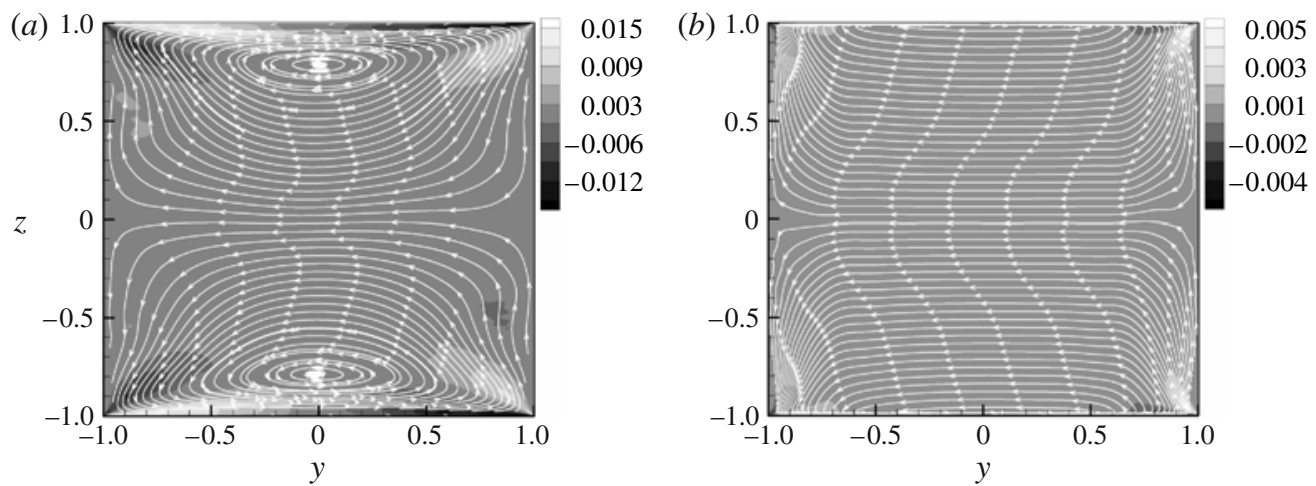

FIGURE 7. Distributions of mean electric current density for the same flows as in figure 3. Contours of the streamwise component $j_{x}$ and projection of streamlines passing through 50 uniformly distributed points on the line $y=0$ (the cross-section $\mathrm{Ha}-\mathrm{Ha}$ ) are shown: (a) $H a=100 ;(b) H a=300$. 

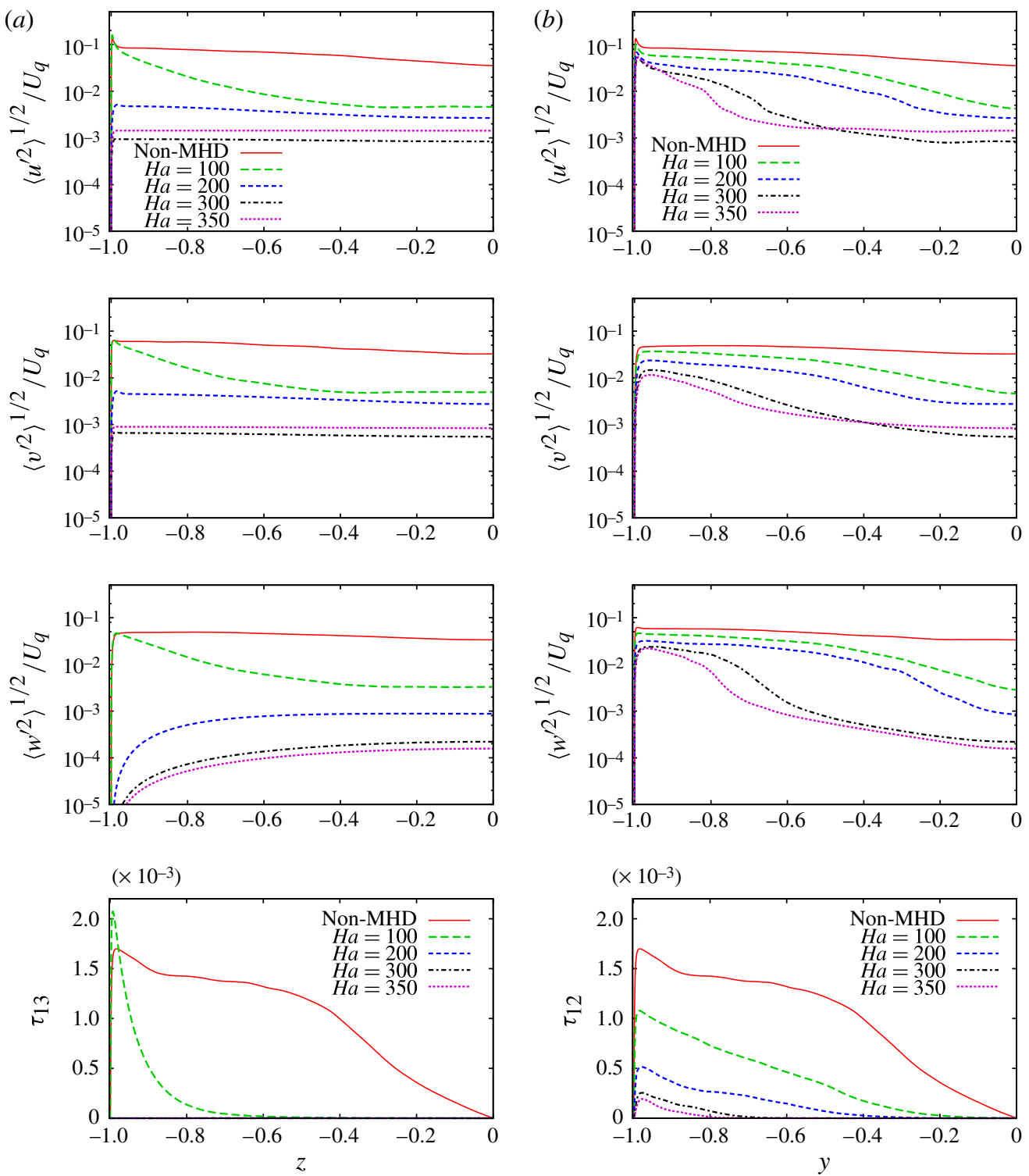

FIgURE 8. (Colour online) Time- and streamwise-averaged r.m.s. velocity fluctuations shown as profiles in the cross-sections (a) Ha-Ha and (b) Sh-Sh. Note that logarithmic scale is used for the r.m.s. values. The Reynolds-stress components $\tau_{13}$ and $\tau_{12}$ corresponding to the wall-normal turbulent transport of streamwise velocity are in the last row.

The first and rather obvious observation is that the imposed magnetic field reduces the average intensity of velocity fluctuations. Furthermore, the profiles are in agreement with the apparent flow structure discussed in $\S 3.1$ (see figure 2). Near the Hartmann walls, strong peaks of the fluctuation amplitude typical for turbulent boundary layers are seen at $H a=100$, but not at higher $H a$. Interestingly, the maximum magnitudes of $\left\langle u^{\prime 2}\right\rangle^{1 / 2},\left\langle v^{\prime 2}\right\rangle^{1 / 2}$ and $\left\langle w^{\prime 2}\right\rangle^{1 / 2}$ are a little larger at $H a=100$ than in the hydrodynamic flow. This can be attributed to the effect of turbulence 
production by stronger mean shear, which overcomes the effect of direct magnetic suppression. This situation is unique among those explored in our study. No turbulence intensities exceeding the intensity at $H a=0$ are observed in the Shercliff or Hartmann layers at $H a>100$. Kobayashi (2008) also does not report such behaviour in the LES at $\operatorname{Re}=14500$.

Near the sidewalls, the turbulent boundary layers exist at all $\mathrm{Ha}$ up to 350 , which is clearly supported by the r.m.s. fluctuation profiles in figure $8(b)$. The intensity of turbulence and the extent of the zone of turbulent activity are smaller at larger $\mathrm{Ha}$.

In the plots of $\tau_{12}$ we see the effect of reduction of the wall-normal turbulent momentum transport due to the suppression of fluctuations by the magnetic field. This appears to be the reason for the reduced slope of the mean velocity profile in the cross-section $S h-S h$ illustrated in figure 5. The same effect has been detected by Krasnov et al. (2008) in a channel with spanwise magnetic field. For $\tau_{13}$, an interesting feature is that at its peak near the Hartmann wall, the transport rate is higher in the MHD flow at $H a=100$ than it is in the flow with $H a=0$. This is evidently related to the similar behaviour of $\left\langle u^{\prime 2}\right\rangle^{1 / 2}$ and $\left\langle w^{\prime 2}\right\rangle^{1 / 2}$ and has the same reason.

A non-trivial feature of the r.m.s. fluctuation profiles in figure 8, which could not be predicted on the basis of the flow visualization in $\$ 3.1$, is that the fluctuation amplitude does not decay to zero in the presumably laminar core zone forming at high Hartmann numbers. The amplitude remains finite, although more than an order of magnitude smaller than the amplitude in the turbulent layers. Analysing the fluctuation amplitudes within this zone, we also find that they are much higher for the components $u^{\prime}$ and $v^{\prime}$ than for the component $w^{\prime}$ parallel to the magnetic field. Another feature is that the amplitudes remain nearly constant across the core in the direction of the magnetic field (the cross-section $\mathrm{Ha}-\mathrm{Ha}$ ). They also do not decrease much with the Hartmann number. In fact, they are somewhat higher for $\mathrm{Ha}=350$ than for $H a=300$. This phenomenon is not reported by Kobayashi (2008), although the profiles of turbulence intensity at $R e=14500, \mathrm{Ha}=58$ appear to indicate weak but finite intensity within the core.

In order to further illustrate the distribution of velocity fluctuations in the flow, including the presence of fluctuations in the core zone, figure 9 shows the turbulent kinetic energy

$$
E=\left\langle u^{\prime 2}\right\rangle+\left\langle v^{\prime 2}\right\rangle+\left\langle w^{\prime 2}\right\rangle
$$

in the flows with $H a=200$ and 300. We see that the energy is, indeed, many orders of magnitude larger than could be created by the round-off error of computer arithmetics. We also see, not unexpectedly, that the energy is virtually uniform along the magnetic field lines.

We conclude that the presumably laminar zone developing between the turbulent Shercliff layers at high $\mathrm{Ha}$ should, in fact, be called 'quasi-laminar', since it contains weak but non-zero fluctuating velocity structures. The structures are dominated by the velocity components orthogonal to the magnetic field and probably extending from one Hartmann wall to another. The nature of these structures will be further investigated in $\S 3.6$.

\subsection{Integral characteristics}

The integral characteristics of the flow are presented in table 2 and figures 10 and 11. We show the centreline velocity $U_{c l}$ of the mean flow, the wall friction Reynolds numbers (2.13) computed using the wall-normal derivatives of the mean velocity along 

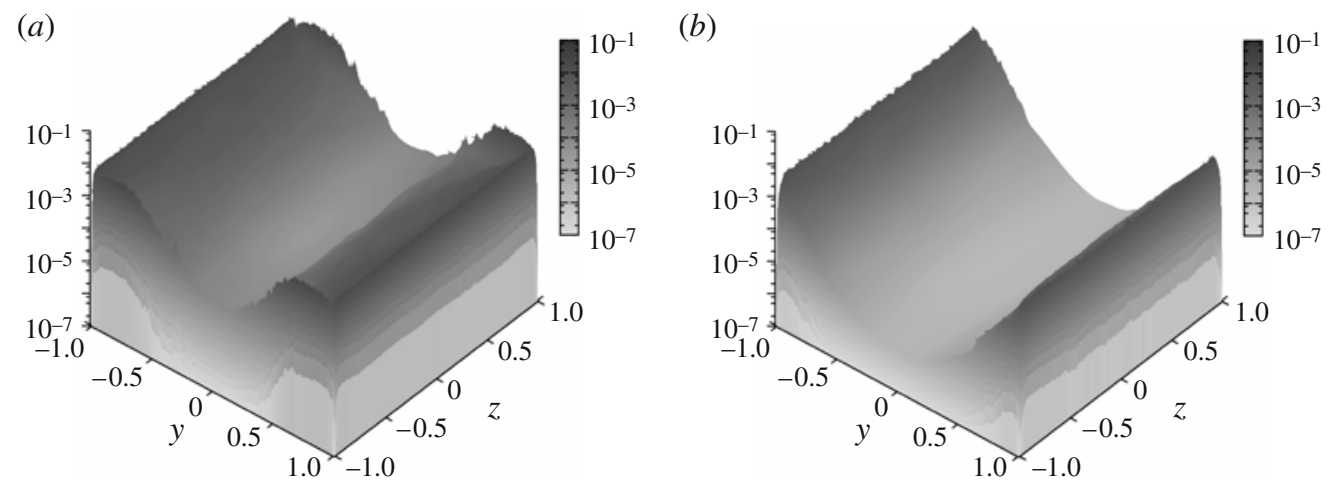

FIGURE 9. Time- and streamwise-averaged turbulent kinetic energy (3.1). The small constant $10^{-7}$ is added to the values of energy to allow plotting in logarithmic coordinates: (a) $H a=200 ;(b) H a=300$.
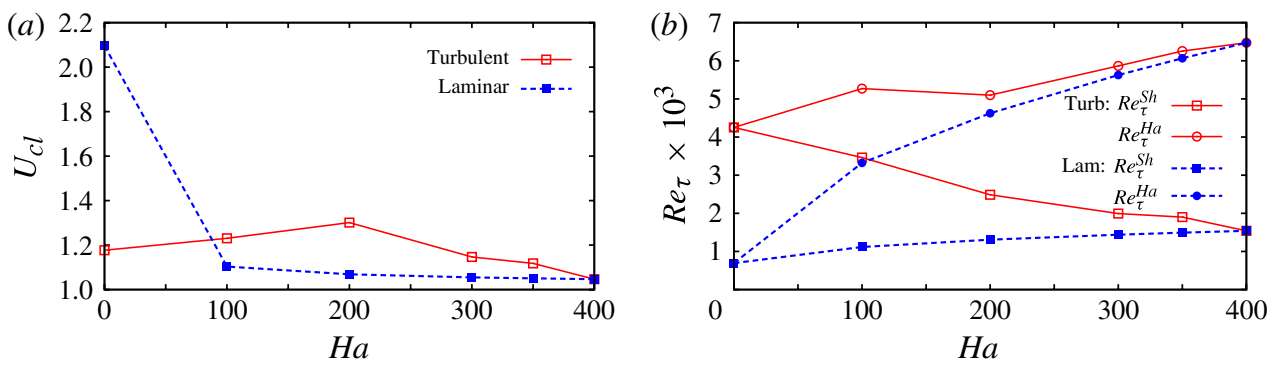

Figure 10. (Colour online) Computed integral characteristics of the flow: $(a)$ centreline mean velocity and $(b)$ wall friction Reynolds numbers (2.13). Data for laminar flows at the same $\mathrm{Ha}$ and $\mathrm{Re}$ are shown for comparison.

the mid-plane cross-sections, and the skin friction coefficients at the Hartmann and Shercliff walls:

$$
C_{f}^{H a}=\frac{1}{R e} \int_{-1}^{1}\left(\left.\frac{\partial U}{\partial z}\right|_{z=-1}-\left.\frac{\partial U}{\partial z}\right|_{z=1}\right) \mathrm{d} y, \quad C_{f}^{S h}=\frac{1}{R e} \int_{-1}^{1}\left(\left.\frac{\partial U}{\partial y}\right|_{y=-1}-\left.\frac{\partial U}{\partial y}\right|_{y=1}\right) \mathrm{d} z .
$$

The friction coefficients can also be expressed as $2 \tau_{w} /\left(\rho U_{q}^{2}\right)$, where $\tau_{w}$ are the average shear stresses measured at the respective walls.

For the purpose of comparison, all the characteristics are presented for the actual computed flow and for the purely laminar flow obtained at the same parameters.

The change of the centreline velocity with $\mathrm{Ha}$ is a manifestation of the variation of the mean flow profile discussed in $\$ 3.2$, which in turn is a result of the modification of the flow turbulence by the magnetic field. We see in figure 10 that $U_{c l}$ changes non-monotonically with $\mathrm{Ha}$. It is significantly larger than in the laminar flow for all the MHD flow states with turbulence.

Predictably, in the flows with magnetic field, the values of the wall-friction Reynolds numbers and friction coefficients are different at the Hartmann and Shercliff walls. The Hartmann friction is significantly larger. Interestingly, $C_{f}^{H a}$ and $R e_{\tau}^{H a}$ change non- 

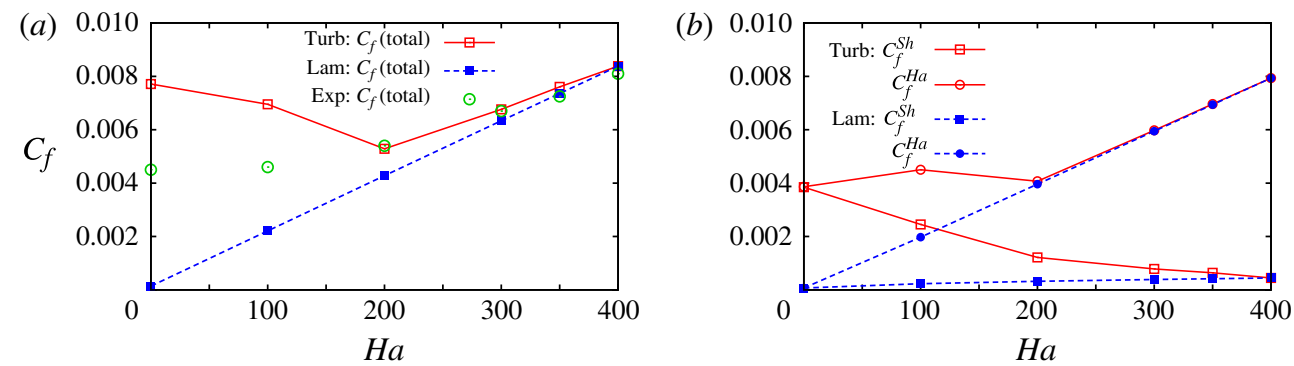

FIGURE 11. (Colour online) Integral friction coefficients (3.2): (a) coefficient of total friction $C_{f}=C_{f}^{S h}+C_{f}^{H a} ;(b)$ coefficients for Shercliff and Hartmann walls. For comparison, data for laminar flows and results of the experiments of Murgatroyd (1953) are shown.

monotonically with $H a$, increasing from $H a=0$ to $H a=100$, then decreasing to $H a=200$, and increasing again at higher $H a$, gradually approaching the laminar flow values. These changes are caused by the combined effects of development of a steeper mean velocity profile within the Hartmann layer and removal of turbulent shear stress by the magnetic field.

For the Shercliff wall coefficients $C_{f}^{S h}$ and $R e_{\tau}^{S h}$, we see a gradual decrease with $\mathrm{Ha}$, which can be explained by gradual suppression of turbulence and hence turbulent momentum transport in the Shercliff layers.

The integral friction coefficient averaged over all four walls of the duct is the flow parameter that can be most reliably measured in experiments. The only experimental data available at the same Reynolds and Hartmann numbers as in our computations are those by Murgatroyd (1953). The correspondence is incomplete, since a duct of crosssection 5:1 was used in the experiment. Therefore we should only expect agreement at high $\mathrm{Ha}$, when the wall friction is dominated by the Hartmann boundary layers. One can see in figure 11 that such agreement is indeed achieved in our simulations. At smaller Hartmann numbers, the relative contribution of friction at the Shercliff walls is significant, and the difference in the duct geometry becomes important, causing a higher friction coefficient in the duct of cross-section $1: 1$, as illustrated by figure 11 . We could attempt to estimate the friction in the 5:1 duct by a linear combination of the Hartmann and Shercliff frictions computed in the 1:1 duct, but the result would inevitably be inaccurate to the extent of defeating the object of comparison with the experiment.

We have also compared with the LES results of Kobayashi (2008) obtained at $R e=14500$ and $H a=0,43.5$ and 58. DNS was performed on a grid with $512 \times 193^{2}$ points, all the other features of the numerical model being the same as in our main computations. The calculated values of $C_{f}$ were in good agreement with those found by Kobayashi (2008).

\subsection{Scaling of mean velocity}

We will now explore whether the mean velocity of the MHD duct flow follows logarithmic or some other scaling law near the walls. Two relevant previous results can be mentioned. A log-layer followed by a plateau extending across the core flow is typical for the Hartmann channel flow (see e.g. Boeck et al. 2007). For channel flow with moderately strong spanwise magnetic field, Krasnov et al. (2008) have found that the log-layer behaviour disappears with growing $\mathrm{Ha}$. 

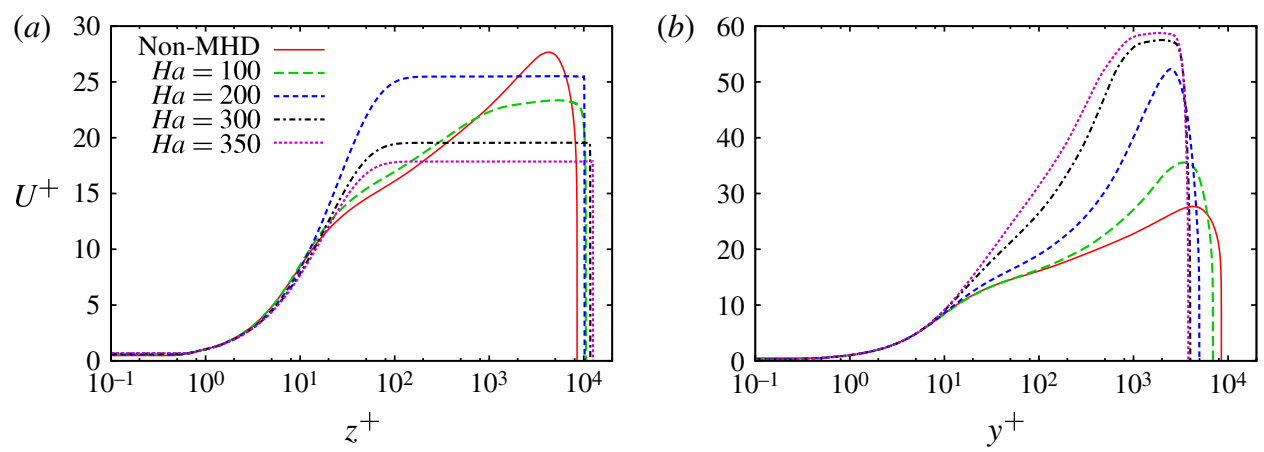

FIGURE 12. (Colour online) Profiles of mean flow velocity plotted in wall units in $(a)$ the mid-plane cross-section $\mathrm{Ha}-\mathrm{Ha}$ and (b) the mid-plane cross-section $\mathrm{Sh}-\mathrm{Sh}$.
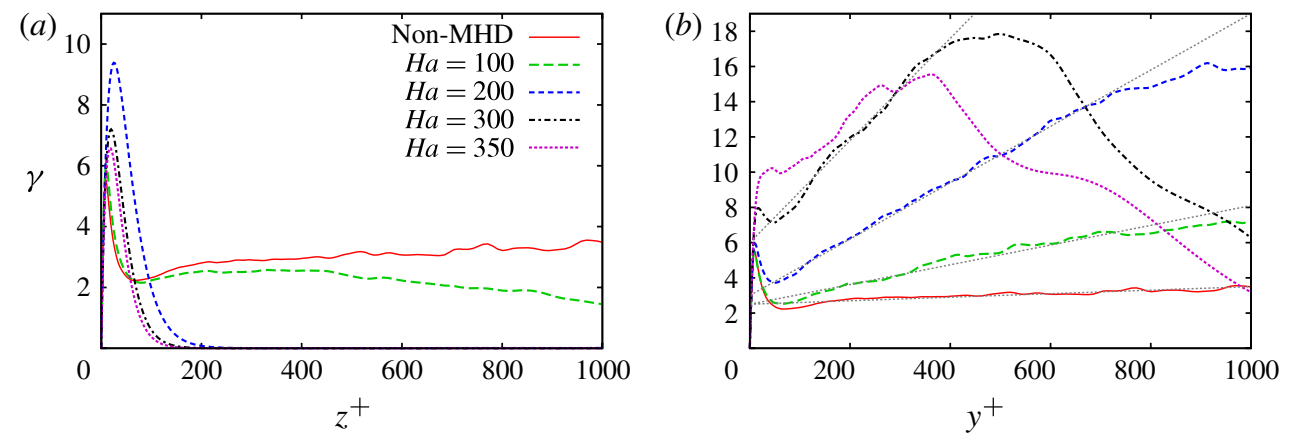

FIgURE 13. (Colour online) Profiles of compensated mean flow velocity gradients (3.5) plotted in the mid-plane cross-sections (a) Ha-Ha and (b) Sh-Sh.

The profiles of the mean streamwise velocity along the lines $H a-H a$ and $S h-S h$ are plotted in wall units in figure 12. The wall units are based on the friction Reynolds numbers $(2.13)$ computed in the respective cross-sections. The coordinates $y^{+}$and $z^{+}$ are defined by (2.14). The mean velocity is scaled as

$$
U^{+}=U / u_{\tau}^{S h} \quad \text { or } \quad U^{+}=U / u_{\tau}^{H a},
$$

where the wall friction velocities are computed as

$$
u_{\tau}^{S h}=R e_{\tau}^{S h} / R e \quad \text { and } \quad u_{\tau}^{H a}=R e_{\tau}^{H a} / R e .
$$

Near the Hartmann walls, we see apparently log-layer behaviour at $H a=0$ and $H a=100$ and profiles typical of a laminar Hartmann layer at higher Ha. Near the Shercliff wall, increasing deviation from log-layer behaviour appears as $\mathrm{Ha}$ increases.

In order to achieve more reliable conclusions, we have calculated the reciprocal von Kármán coefficients as compensated gradients of mean velocity

$$
\gamma=z^{+} \frac{\partial U^{+}\left(y=0, z^{+}\right)}{\partial z^{+}} \quad \text { and } \quad \gamma=y^{+} \frac{\partial U^{+}\left(y^{+}, z=0\right)}{\partial y^{+}} \text {. }
$$

A sizeable range of $y^{+}$or $z^{+}$, in which $\gamma$ is nearly constant, is an indication of log-layer behaviour.

The results are presented in figure 13. We see that the turbulent layer near the Hartmann wall at $\mathrm{Ha}=100$ has a log-layer, which is about as wide as in the flow 

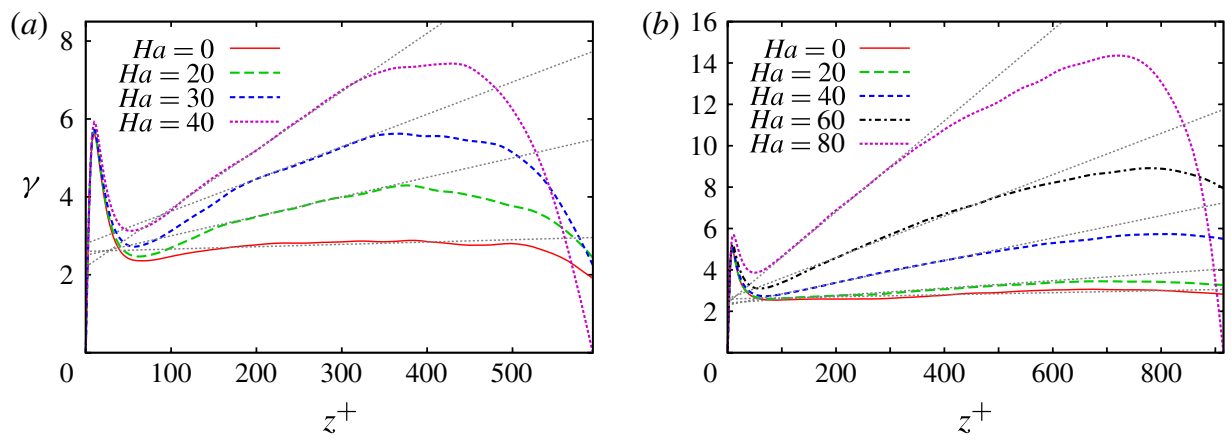

FIGURE 14. (Colour online) Compensated mean velocity gradient for the turbulent channel flow under spanwise magnetic field. (a) The results of Krasnov et al. (2008) obtained in DNS at $R e=13333 ;(b)$ the results of LES at $R e=26666$.

at $H a=0$. The results for the Shercliff layer demonstrate a different and rather striking tendency. No extended regions of approximately constant $\gamma$ can be found. Instead, $\gamma$ can be approximated by a linear function

$$
\gamma=\gamma_{0}+\gamma_{1} y^{+} .
$$

The approximation is quite accurate in wide ranges of $y^{+}$at $H a=100,200$ and 300. The results for $H a=350$ are inconclusive, primarily because of the relatively small thickness of the turbulent layer.

Upon re-examination of the DNS and LES results of Krasnov et al. (2008) for channel flow in a spanwise magnetic field, we have also found linear portions of the profiles of compensated velocity gradients at $R e=6666$ and 13333. This is illustrated for $R e=13333$ in figure 14. Also included in the figure and showing the same behaviour are the results of the dynamic Smagorinsky LES of the channel flow at $R e=26666$ conducted especially for this paper. The LES were performed using the same pseudo-spectral solver as in Krasnov et al. (2008). The computational domain was $2 \pi \times \pi \times 2$ and the grid size was $256^{3}$ collocated points. The time-averaged data were obtained in calculations of fully developed flows for 100 convective time units.

The reciprocal von Kármán coefficient in the form of (3.6) can be interpreted as an indicator of combined log-layer (the constant $\gamma_{0}$ ) and linear behaviour of the mean velocity. The $\log$-layer behaviour dominates at small $y^{+}$, more precisely at $y^{+} \ll \gamma_{0} / \gamma_{1}$. In the limit $y^{+} \gg \gamma_{0} / \gamma_{1}$, substituting (3.6) into the second equation of (3.5) we find

$$
\frac{\partial U^{+}\left(y^{+}, z=0\right)}{\partial y^{+}} \approx \gamma_{1} .
$$

It has to be noted that, according to our computations shown in figures 13 and $14, \gamma_{0}$ is two or three orders of magnitude larger than $\gamma_{1}$, so the large $y^{+}$limit (3.7) is never fully attained.

The possibility of a linear velocity profile is not a complete novelty. It has been detected previously, in particular in the flow in a rotating channel (Johnston, Haleen \& Lezius 1972). In a similar way, we can apply simple arguments to derive a scaling model for the slope $\gamma_{1}$. The starting point is the assumption that the slope is inversely proportional to the relevant time scale, which in our case is the Joule dissipation time 
$\tau_{J} \equiv \rho / \sigma B^{2}:$

$$
\frac{\partial U(y, z=0)}{\partial y} \sim \frac{1}{\tau_{J}} .
$$

Replacing $U$ and $y$ by velocity and distance in wall units, we find

$$
\frac{u_{\tau}^{2}}{v} \frac{\partial U^{+}}{\partial y^{+}} \sim \frac{1}{\tau_{J}}
$$

which leads to

$$
\gamma_{1}=\frac{\partial U^{+}}{\partial y^{+}} \sim \frac{v^{2}}{u_{\tau}^{2}} \frac{\sigma B^{2}}{\rho \nu}=\frac{H a^{2}}{\left(R e_{\tau}^{S h}\right)^{2}} .
$$

An attempt to verify the scaling is presented in figure 15 , where $\gamma_{1}$ is shown as a function of $\mathrm{Ha}^{2} /\left(\operatorname{Re}_{\tau}^{S h}\right)^{2}$. The data for the channel flow with spanwise magnetic field are also included. We see that the scaling is fairly accurately followed by the channel flow, as indicated by the straight dotted line through the origin. It is a visual fit to the relation (3.10). The coefficient computed for the duct flow follows the trend at $H a=100$ and 200, but appears to deviate from it at $H a=300$ (the rightmost point in figure 15). One possible explanation for the deviation is the small thickness of the layer with log-lin behaviour at this $H a$ (see figure 13), which can lead to inaccurate estimate of $\gamma_{1}$. Another reason is that the steepening of the duct basic profile is observed in a limited range of $\mathrm{Ha}$, contrary to its channel counterpart. Therefore, the associated log-layer behaviour is also not expected to be clearly pronounced, as the flow starts to converge to the laminar flat profile of the MHD duct. For our simulations this limit can be identified as $H a \approx 200$.

\subsection{Turbulence at high $\mathrm{Ha}$}

In this section, we apply additional visualizations and diagnostics in order to analyse the internal structure of the turbulent flows. The main attention is given to cases of a strong magnetic field.

Figure 16 shows the instantaneous distribution of isosurfaces of the intermediate eigenvalue $\lambda_{2}$ of the tensor $S_{i k} S_{k j}+\Omega_{i k} \Omega_{k j}$, where $S_{i j} \equiv\left(u_{i, j}+u_{j, i}\right) / 2$ and $\Omega_{i j} \equiv$ $\left(u_{i, j}-u_{j, i}\right) / 2$. As shown by Jeong \& Hussein (1995), connected regions of negative $\lambda_{2}$ are indicators of coherent vortical structures.

We see that each turbulent layer near a Shercliff wall can be divided into two zones with rather different natures of turbulent fluctuations. Closer to the wall, turbulence is dominated by small-scale structures without apparent anisotropy. Farther from the wall, in a sub-layer sandwiched between the zone of small-scale turbulence and the quasi-laminar core, the flow is dominated by large vortices. The vortices are strongly distorted but can still be identified as columnar vortices with axes approximately aligned with the magnetic field. Such an anisotropic but not purely two-dimensional state is familiar from the studies of homogeneous MHD turbulence, for example those of Zikanov \& Thess (1998) and Vorobev et al. (2005), where it has been found to occur at $N \sim 1$. As discussed by Thess \& Zikanov (2007), the main source of the distortion is likely to be the elliptic instability.

A good measure of the structural anisotropy of the flow (the directional disparity of the typical length scales) is the set of coefficients

$$
G_{i j}^{k} \equiv \frac{\left\langle\left(\partial u_{k} / \partial x_{i}\right)^{2}\right\rangle\left(1+\delta_{k i}\right)}{\left\langle\left(\partial u_{k} / \partial x_{j}\right)^{2}\right\rangle\left(1+\delta_{k j}\right)}, \quad i \neq j .
$$




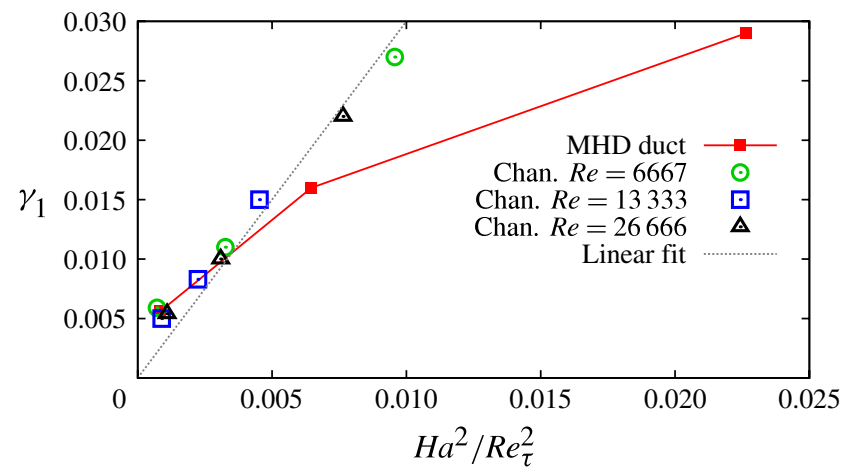

FIGURE 15. Verification of scaling (3.10). The slope $\gamma_{1}$ of the linear portion of the compensated mean velocity profiles is shown for the duct flow and for the channel flow with spanwise magnetic field. The results for the duct flow are plotted versus $H a^{2} /\left(R e_{\tau}^{S h}\right)^{2}$.

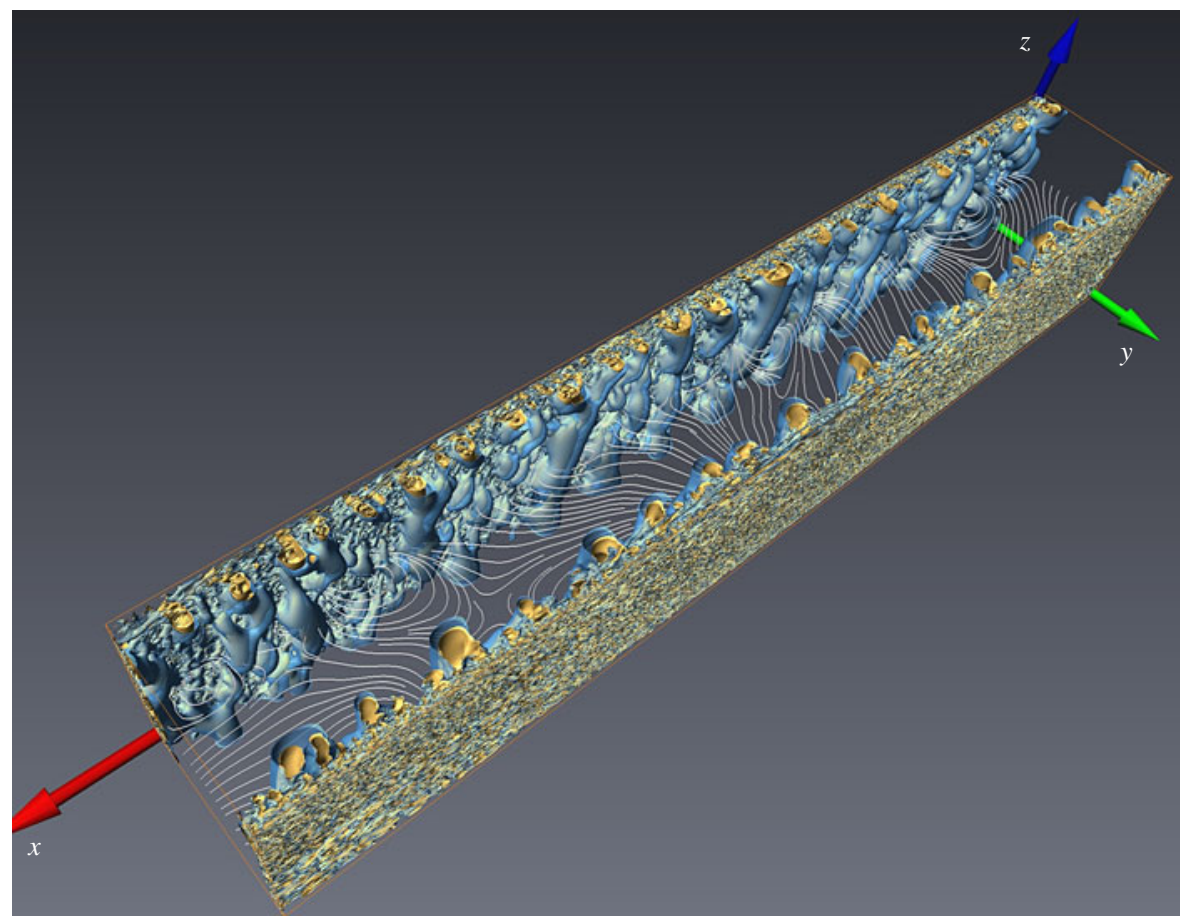

FIGURE 16. Instantaneous distribution of coherent vortices shown as isosurfaces $-0.01 \lambda_{2}^{r m s}$ (yellow) and $-0.005 \lambda_{2}^{r m s}$ (cyan) of the second eigenvalue $\lambda_{2}$ of tensor $S_{i k} S_{k j}+\Omega_{i k} \Omega_{k j}$. Also shown are the streamlines of velocity fluctuations in the mid-plane $S h-S h$.

Each coefficient should have a value of about one in a flow with isotropic distribution of the respective velocity component. The coefficients should be zero in purely twodimensional flows uniform in the direction of the coordinate $x_{i}$. The coefficients have been applied to MHD turbulence before, for example by Zikanov \& Thess (1998), Kassinos, Knaepen \& Wray (2006) and Krasnov et al. (2008). In the case of MHD 

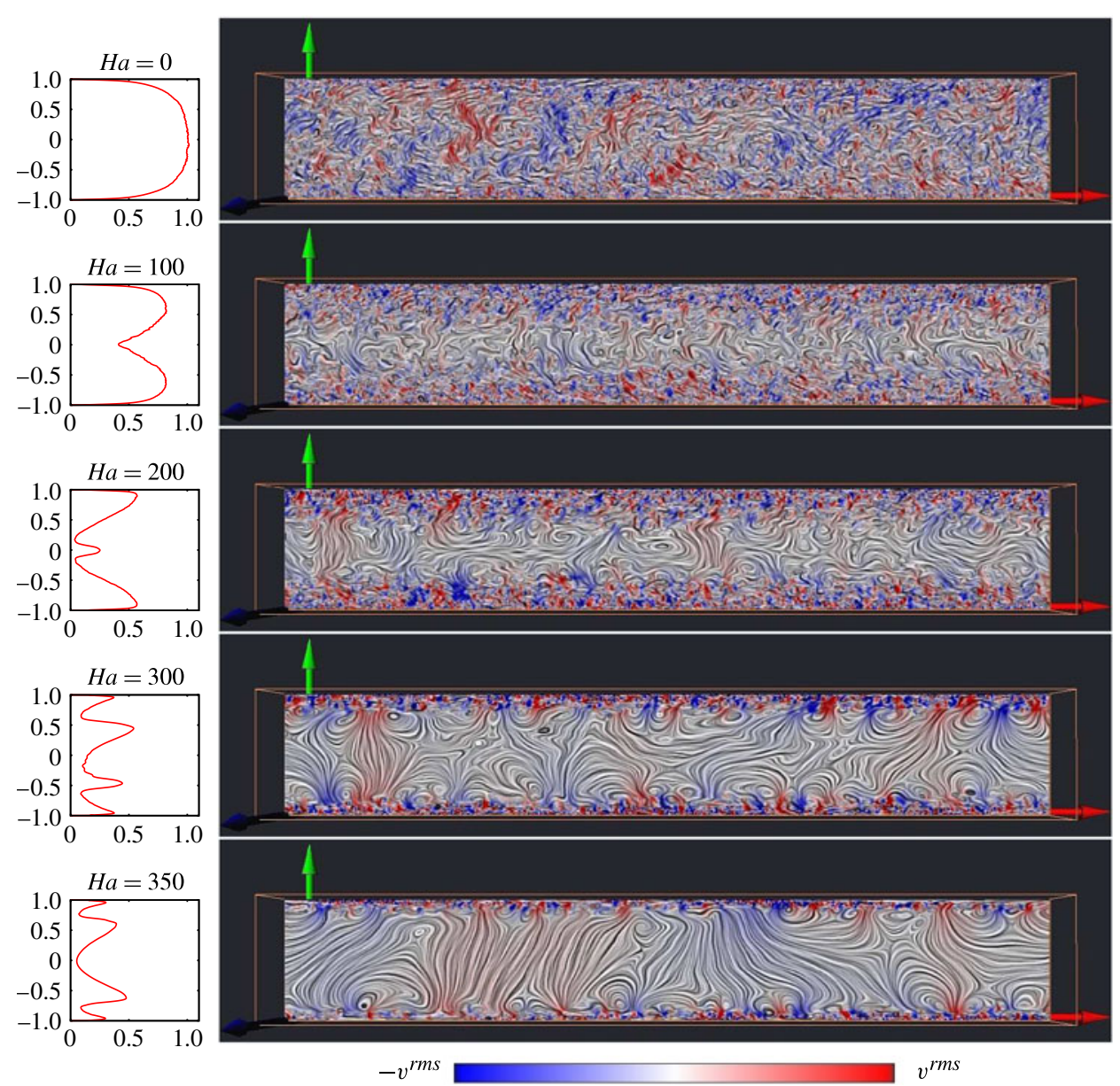

FIGURE 17. Visualizations of flow streamlines in the entire domain (planar linear integral convolution curves in the mid-plane $S h-S h$ ) computed on the basis of instantaneous velocity fields. Profiles of the anisotropy coefficient $G_{32}^{3}$ (see (3.12)) in the mid-plane $S h-S h$ are also shown. The colour gradient corresponds to the spanwise velocity $v$ and changes within the limits of $\pm v^{r m s}$ for each set of parameters.

duct flow, there are two sources for the structural anisotropy. It can be caused by the mean shear, which leads to elongation of flow structures in the streamwise direction, and by the magnetic field, which elongates the structures along the field lines.

We select a single coefficient, namely

$$
G_{32}^{3} \equiv \frac{2\left\langle(\partial w / \partial z)^{2}\right\rangle}{\left\langle(\partial w / \partial y)^{2}\right\rangle},
$$

for the presentation. The choice is determined by our primary interest in the effect of the magnetic field. Since the coefficient excludes $x$-derivatives and is based on the $w$ velocity components, it appears to be a particularly good measure of the elongation of flow structures in the $z$-direction shown in figure 16 .

The results are presented in figure 17, which pairs the profiles of $G_{32}^{3}$ along the cross-section $S h-S h$ with the projections of streamlines on the same cross-section. 
Instantaneous velocity distributions are used to generate the streamlines and to evaluate $G_{32}^{3}$. This means that $\langle\cdots\rangle$ in (3.12) now stands for the averaging in $x$. The streamlines are drawn using the method of planar linear integral convolution (Cabral \& Leedom 1993), which allows one to use all velocity points and to avoid uncertainty related to the choice of seeding points in the traditional streamline generation techniques.

The results are especially interesting in cases of a strong magnetic field $(\mathrm{Ha}=300$ and 350). The anisotropy coefficient has local peaks not far from Shercliff walls, where the flow has small-scale three-dimensional turbulent fluctuations. The peak values of $\sim 0.4$ indicate that the fluctuations are substantially anisotropic, although far from two-dimensional. Farther from the walls, where the dominating structures are the large-scale distorted columnar vortices, the anisotropy coefficient decreases to approximately 0.1 and then increases to 0.4 or 0.5 . Correlating the behaviour with the visible flow structure, we conclude that the local minima of $G_{32}^{3}$ correspond to the strongly anisotropic axes of the large-scale vortices. The inner peaks correspond to the areas where the vortices are strongly deformed by the elliptic instability.

Figure 17 provides a good illustration of the nature of velocity fluctuations in the quasi-laminar core. The strength of the flow is illustrated by colouring the streamlines by the local amplitude of the velocity component $v$. We see that the streamlines extend through the entire core from one turbulent Shercliff wall layer to the other. The flow is weak and tends to form large zones of transport in the same direction. We also see that the anisotropy coefficient decreases but does not become zero, indicating a state with velocity nearly - but not entirely - uniform along the magnetic field lines. The flow within the core can be described as a weak nearly two-dimensional motion controlled by the columnar vortices on both sides.

\section{Conclusion}

In conclusion, we summarize the main results of the work just presented. It appears that, when the Reynolds number is not small, states with a laminar core and turbulent Shercliff layers must be considered as typical, rather than exceptional, states of the MHD duct flow. In our study, the range of $\mathrm{Ha}$ corresponding to such states is broader than the range of fully turbulent regimes. The range decreases at small $R e$, as indicated by our DNS at $R e=5000$ (Krasnov et al. 2011b) and LES at $R e=14500$ (Kobayashi 2008).

The Shercliff layer turbulence has peculiar characteristics, including the separation into sub-layers of small-scale and large-scale structures and the log-linear scaling. We also find that the apparently laminar core should be properly called quasi-laminar, since weak but non-negligible velocity fluctuations are created in it by the circulation induced by the large-scale Shercliff layer vortices.

In a broad sense, the applicability of our results is not limited to the flow in a duct of square cross-section. The mechanisms defining the flow structure are the same in the high-Re flows in ducts of other cross-sections and pipes and thus similar structure must be expected.

In the future, it would be interesting to conduct a detailed analysis of the statistical properties of turbulent fluctuations, such as energy spectra and velocity two-point correlations and structure functions. Such a study would further help us to understand the flow transformation under the combined action of mean shear and magnetic field and complement the studies of properties of homogeneous turbulence, such as Zikanov \& Thess (1998), Kassinos et al. (2006), Burattini, Zikanov \& Knaepen (2010) and Favier et al. (2011). 


\section{Acknowledgements}

We are thankful to M. Rossi, M. Uhlmann and B. Knaepen for interesting discussions and useful comments. T.B. and D.K. acknowledge financial support from the Deutsche Forschungsgemeinschaft (grant Bo 1668/5). O.Z. acknowledges financial support from the Deutsche Forschungsgemeinschaft (Graduiertenkolleg GRK 1567) and from the US National Science Foundation (grant CBET 0966557). Computer resources were provided by the computing centre of the Forschungszentrum Jülich (NIC). Visualization and post-processing of the results was performed at the computing centre of TU Ilmenau.

\section{REFERENCES}

Adams, J. C., Swarztrauber, P. \& Sweet, R. 1999 Efficient Fortran subprograms for the solution of separable elliptic partial differential equations. http://www.cisl.ucar.edu/css/software/ fishpack/.

BAYLIS, J. A. 1971 Experiments on laminar flow in curved channels of square section. J. Fluid Mech. 48 (3), 417-422.

Boeck, T., Krasnov, D. \& Zienicke, E. 2007 Numerical study of turbulent magnetohydrodynamic channel flow. J. Fluid Mech. 572, 179.

BRANOVER, H. 1978 Magnetohydrodynamic Flow in Ducts. Wiley.

Brouillette, E. C. \& Lykoudis, P. S. 1967 Magneto-fluid-mechanic channel flow. Part 1. Experiment. Phys. Fluids 10 (5), 995-1001.

Burattini, P., Zikanov, O. \& Knaepen, B. 2010 Decay of magnetohydrodynamic turbulence at low magnetic Reynolds number. J. Fluid Mech. 657, 502-538.

Burr, U., Barleon, L., Müller, U. \& Tsinober, A. 2000 Turbulent transport of momentum and heat in magnetohydrodynamic rectangular duct flow with strong sidewall jets. J. Fluid Mech. 406, 247-279.

CABRAL, B. \& LeEDOM, L. 1993 Imaging vector fields using line integral convolution. In Proceedings of the SIGGRAPH '93, Computer Graphics 27, Annual Conference Series, pp. 263-272. ACM SIGGRAPH.

Chaudhary, R., VAnKa, S. P. \& Thomas, B. G. 2010 Direct numerical simulations of magnetic field effects on turbulent flow in a square duct. Phys. Fluids 22 (7), 075102.

Davidson, P. A. 2001 An Introduction to Magnetohydrodynamics. Cambridge University Press.

DEY, P. \& ZIKANOV, O. 2012 Turbulent flow and transport of passive scalar in magnetohydrodynamic channel flows with different orientations of magnetic field. Intl J. Heat Fluid Flow (in press).

Favier, B., Godeferd, F. S., Cambon, C., Delache, A. \& Bos, W. J. T. 2011 Quasi-static magnetohydrodynamic turbulence at high Reynolds number. J. Fluid Mech. 681, 434-461.

Galletti, B. \& Bottaro, A. 2004 Large-scale secondary structures in duct flow. J. Fluid Mech. 512, 85-94.

GAVRILAKIS, S. 1992 Numerical simulation of low-Reynolds-number turbulent flow through a straight square duct. J. Fluid Mech. 244, 101-129.

HARTMANN, J. $1937 \mathrm{Hg}$-dynamics I: theory of the laminar flow of an electrically conductive liquid in a homogeneous magnetic field. K. Dan. Vidensk. Selsk. Mat. Fys. Medd. 15 (6), 1-28.

HartmanN, J. \& LAZARUS, F. $1937 \mathrm{Hg}$-dynamics II: experimental investigations on the flow of mercury in a homogeneous magnetic field. K. Dan. Vidensk. Selsk. Mat. Fys. Medd. 15 (7), $1-45$.

HoyAs, S. \& JimÉnEZ, J. 2006 Scaling of the velocity fluctuations in turbulent channels up to $\operatorname{Re}_{\tau}=2003$. Phys. Fluids 18, 011702.

JeOng, J. \& Hussein, F. 1995 On the identification of a vortex. J. Fluid Mech. 285 (1), 69-94.

Johnston, J. P., Haleen, R. M. \& Lezius, D. K. 1972 Effect of the spanwise rotation on the structure of two-dimensional fully developed turbulent channel flow. J. Fluid Mech. 56 (3), 533-557. 
Kassinos, C., Knaepen, B. \& Wray, A. 2006 The structure of MHD turbulence subjected to mean shear and frame rotation. J. Turbul. 7 (1).

Knaepen, B., Kassinos, S. \& CARATi, D. 2004 Magnetohydrodynamic turbulence at moderate magnetic Reynolds number. J. Fluid Mech. 513, 199-220.

KobAYASHI, H. 2006 Large eddy simulation of magnetohydrodynamic turbulent channel flows with local subgrid-scale model based on coherent structures. Phys. Fluids 18, 045107.

Kobayashi, H. 2008 Large eddy simulation of magnetohydrodynamic turbulent duct flows. Phys. Fluids 20, 015102.

Krasnov, D., Zikanov, O. \& Boeck, T. $2011 a$ Comparative study of finite difference approaches to simulation of magnetohydrodynamic turbulence at low magnetic Reynolds number. Comput. Fluids 50, 46-59.

Krasnov, D., Zikanov, O., Rossi, M. \& Boeck, T. 2010 Optimal linear growth in magnetohydrodynamic duct flow. J. Fluid Mech. 653, 273-299.

Krasnov, D., Zikanov, O., Schumacher, J. \& Boeck, T. 2008 Magnetohydrodynamic turbulence in a channel with spanwise magnetic field. Phys. Fluids 20 (9), 095105.

Krasnov, D., Zikanov, O., Thess, A. \& Boeck, T. $2011 b$ Direct numerical simulation of transition and turbulence in MHD duct flow. In The 8th PAMIR Conference on Fundamental and Applied MHD, pp. 157-161. Borgo.

Krasnov, D. S., Zienicke, E., Zikanov, O., Boeck, T. \& Thess, A. 2004 Numerical study of instability and transition to turbulence in the Hartmann flow. J. Fluid Mech. 504, 183-211.

LEE, D. \& CHOI, H. 2001 Magnetohydrodynamic turbulent flow in a channel at low magnetic Reynolds number. J. Fluid Mech. 429, 367-394.

Moresco, P. \& AlboussiÈre, T. 2004 Experimental study of the instability of the Hartmann layer. J. Fluid Mech. 504, 167-181.

Morinishi, Y., Lund, T. S., Vasilyev, O. V. \& Moin, P. 1998 Fully conservative higher order finite difference schemes for incompressible flow. J. Comput. Phys. 143, 90-124.

MÜlleR, U. \& BÜHLER, L. 2001 Magnetohydrodynamics in Channels and Containers. Springer.

Murgatroyd, W. 1953 Experiments on magnetohydrodynamic channel flow. Phil. Mag. 44, $1348-1354$.

Ni, M.-J., Munipalli, R., Huang, P., Morley, N. B. \& Abdou, M. A. 2007 A current density conservative scheme for incompressible MHD flows at a low magnetic Reynolds number. Part 1. On a rectangular collocated grid system. J. Comput. Phys. 227, 174-204.

Potherat, A. 2007 Quasi-two-dimensional perturbations in duct flows under transverse magnetic field. Phys. Fluids 19, 074104.

Potherat, A. \& SCHWEItzer, J.-P. 2011 A shallow water model for magnetohydrodynamic flows with turbulent Hartmann layers. Phys. Fluids 23 (5), 055108.

Potherat, A., Sommeria, J. \& Moreau, R. 2000 An effective two-dimensional model for MHD flows with transverse magnetic field. J. Fluid Mech. 424, 75-100.

Reed, C. B. \& LyKoudis, P. S. 1978 The effect of a transverse magnetic field on shear turbulence. J. Fluid Mech. 89, 147-171.

SARris, I. E., KASsinos, S. C. \& CARATI, D. 2007 Large-eddy simulations of the turbulent Hartmann flow close to the transitional regime. Phys. Fluids 19 (8), 085109.

Satake, S., Kunugi, T., TAKase, K. \& OSE, Y. 2006 Direct numerical simulation of turbulent channel flow under a uniform magnetic field for large-scale structures at high Reynolds number. Phys. Fluids 18 (12), 125106.

Shatrov, V. \& Gerbeth, G. 2010 Marginal turbulent magnetohydrodynamic flow in a square duct. Phys. Fluids 22, 084101.

Sommeria, J. \& Moreau, R. 1982 Why, how and when MHD-turbulence becomes twodimensional. J. Fluid Mech. 118, 507-518.

Thess, A. \& ZiKanov, O. 2007 Transition from two-dimensional to three-dimensional magnetohydrodynamic turbulence. J. Fluid Mech. 579, 383-412.

Uhlmann, M., Pinelli, A., Kawahara, G. \& Sekimoto, A. 2007 Marginally turbulent flow in a square duct. J. Fluid Mech. 588, 153-162.

Vorobev, A., Zikanov, O., Davidson, P. A. \& Knaepen, B. 2005 Anisotropy of magnetohydrodynamic turbulence at low magnetic Reynolds number. Phys. Fluids 17 (12), 125105. 
ZHAO, Y. \& ZIKANOV, O. 2012 Instabilities and turbulence in magnetohydrodynamic flow in a toroidal duct prior to transition in Hartmann layers. J. Fluid Mech. 692, 288-316.

ZhaO, Y., ZiKANOV, O. \& KRASNOV, D. 2011 Instability of magnetohydrodynamic flow in an annular channel at high Hartmann number. Phys. Fluids 23, 084103.

ZIKANOV, O. \& Thess, A. 1998 Direct numerical simulation of forced MHD turbulence at low magnetic Reynolds number. J. Fluid Mech. 358, 299-333. 